\title{
Masked singleton effects
}

\author{
BJÖRN HELD \\ Birkbeck College, University of London, London, England \\ ULRICH ANSORGE \\ Universität Wien, Vienna, Austria \\ and Universität Osnabrück, Osnabrück, Germany \\ AND \\ Hermann J. Müller \\ Birkbeck College, University of London, London, England \\ and Ludwig-Maximilians-Universität München, Munich, Germany
}

\begin{abstract}
In the present study, we tested whether visual singletons remaining outside awareness are processed. Singletons differ by at least one feature from their more homogeneous neighbors. Here, we used backward masking to prevent awareness of shape singleton primes (Experiments 1-4) or color singleton primes (Experiment 5). Masked singleton primes nonetheless produced a congruence effect: Congruent singletons indicating the location of subsequent visible targets facilitated responses, in comparison with incongruent singletons at positions away from the targets. This congruence effect was found with singletons defined by feature presence or feature absence. It was at least partly due to attentional capture by singletons remaining outside of the participants' awareness. Results are discussed in light of theories pertaining to singleton effects and masked-priming effects.
\end{abstract}

Imagine that you are correcting a manuscript for typos. Spotting an erroneous blank space is easy if the additional blank is located between letters within a word. However, it is fairly difficult to find if it is located between words. One factor that contributes to this asymmetry in visual search difficulty is singleton capture. Many studies have confirmed that visual stimuli that differ by one or several features from their more homogeneous neighbors capture attention (for reviews, see Burnham, 2007; Theeuwes, 1992, 2004). Van Zoest, Donk, and Theeuwes (2004), for example, found that a right-tilted bar among several vertical bars captured attention. Accordingly, in our example above, a blank space between two flanking letters within a word captures attention more easily and can be spotted better than, for example, a blank space flanked by another blank space on one side and a letter on the other.

According to Bacon and Egeth (1994), attentional capture by a singleton is goal dependent. Only if a participant is set to search for a singleton will a singleton capture attention. These authors observed that for attentional capture by an irrelevant color singleton to occur, participants had to search for a shape singleton. If one green circle among several green diamonds had to be searched for as a (shape) singleton (because it contained the response-relevant target line), a red diamond as an irrelevant color singleton captured attention. However, if several shape singletons (e.g., a circle, a triangle, and an octagon) were presented at the same time, participants had to change search mode: To find the target by searching for a (shape) singleton was no longer sufficient; rather, participants now had to search for a specific shape (e.g., a circle). In line with a goaldependent effect, under these conditions, the irrelevant color singleton failed to capture attention.

By contrast, Theeuwes $(1992,2004)$ and others (e.g., Burnham \& Neely, 2008) have regarded singleton capture as being stimulus driven in nature. On this view, any strong local feature difference has the power to attract attention (Bergen \& Julesz, 1983; Burnham \& Neely, 2008). This position fits well with results from eyetracking studies: Participants freely viewing 2-D images of natural scenes tend to fixate on strong local feature differences in color, luminance, or orientation (see Itti \& Koch, 2001; Parkhurst, Law, \& Niebur, 2002).

In the present study we tested one prediction of computational theories concerning the role of awareness for singleton capture. Computational theories consider singleton capture to be a consequence of objective feature differences: Typically, an image location's potential to attract or capture attention is assessed as its pooled or summed standard deviation across several visual dimensions (e.g., color, orientation, luminance). This aggregated feature difference is then "compared" with similarly calculated standard deviations at other image locations (see Itti \& Koch, 2001; Parkhurst et al., 2002). The image location 
with the highest deviation has maximal potential to attract attention. The lower the feature deviation at a particular location, the less likely it is that it will attract attention. Crucially, these theories do not assume the viewer's subjective or phenomenal awareness of a feature contrast (or singleton) as in any way crucial for its potential to capture attention. All that is required for singleton capture is a strong, objective feature difference.

Although it is clear that awareness of stimulus salience - that is, literally seeing one stimulus popping out among the others-is not the same thing as a singleton effect (see Yantis \& Egeth, 1999), whether awareness of a singleton is or is not a crucial prerequisite of a singleton effect has rarely been studied. A number of studies have investigated whether abrupt-onset singletons (presented asynchronously with all the other stimuli) can remain outside participants' awareness and still capture attention (see McCormick, 1997; Scharlau, 2002). Yet abrupt-onset singletons are special (Cole, Kentridge, \& Heywood, 2005).

Our review of the literature revealed a single study that seemed to have investigated whether stimulus awareness is a crucial precondition for a singleton effect in healthy human volunteers. Zhaoping (2008) used dichoptic stimulation. In congruent conditions, an image containing a slightly tilted bar, the response-relevant orientation target, was presented to one eye only, and a background of horizontally aligned, homogeneous distractors was presented to the other eye. By contrast, in incongruent conditions, the tilted target was shown together with all but one horizontal distractor to one eye, and the one, "missing" horizontal distractor was shown to the other eye. Although the participants were unaware of this eye-of-origin singleton distractor in the incongruent conditions, this singleton captured attention: Search for and responses to the target were facilitated in the congruent condition when the singleton distractor was presented on the same side as the target (as compared with a singleton distractor, presented on the side opposite the target). This capture effect by an eye-of-origin singleton would argue for a salience effect originating in (or even below) V1. In addition and in contrast to Itti and Koch's (2001) feature contrast explanation, Zhaoping (2008) argued that the salience of actually present features, represented in V1, rather than mere feature contrast, was responsible for awareness-independent capture effects (see Li, 2002).

Our aim in the present study was to examine whether Zhaoping's (2008) results would also extend to more natural viewing conditions and could indeed be due to feature salience. Although her study pinpointed salience effects arising in (or below) V1, single-eye-defined singletons do not play a major role for capture under natural viewing conditions in which visual stimuli impinge on both eyes - which is why salience defined by eye of origin is not normally accommodated within computational models of contrast-driven capture. Given this, we asked more generally whether awareness-independent singleton effects would also be found with singletons defined by visual image features, such as orientation (Experiments 1-4) and color (Experiment 5).
For this purpose, we used backward masking (see Breitmeyer \& Ogmen, 2006; Klotz \& Neumann, 1999) of singletons by surrounding metacontrast masks. Presenting a visual stimulus only briefly, followed by a closely surrounding backward mask, can drastically lower stimulus visibility and participants' awareness of a singleton (see Breitmeyer, 1984; Neumann \& Klotz, 1994). At the same time, backward masking leaves responding to masked stimuli (see Fehrer \& Raab, 1962; Klotz \& Neumann, 1999; Vorberg, Mattler, Heinecke, Schmidt, \& Schwarzbach, 2003) and attending to them (see Mulckhuyse, Talsma, \& Theeuwes, 2007; Steglich \& Neumann, 2000) largely unaffected.

\section{EXPERIMENT 1}

To test whether a masked orientation singleton is processed, we used the following procedure. In a target choice response task (CRT), we instructed our participants to respond to one visible target singleton. The target differed by two bars from the other stimuli in the display. As can be seen in Figure 1 (lower panel), three clearly visible boxesone left, one right, and one central-were presented on each trial. The participants had to press a key (left or right) on that side on which a pair of bars flanked the target box.

In addition to the clearly visible target bars and boxes, small boxes at all three positions were presented as primes. Primes preceded the larger target bars and boxes. One prime stimulus was also a singleton: The prime singleton consisted of a small pair of bars flanking only one of the prime boxes. Crucially, the prime display was masked: The surrounding boxes of the target display and the brief interval between the prime and target ensured metacontrast masking of the primes by the target boxes (see Breitmeyer, 1984; Breitmeyer \& Ogmen, 2006).

For our test of the singleton effect, the prime singleton was presented either at the same position as the visible target singleton (congruent condition) or on the side opposite the target singleton (incongruent condition). In the congruent condition, if the prime singleton captured attention, attention would be allocated in advance to the position of the upcoming singleton target and should, thus, facilitate responding to the target (see Jonides, 1981; Müller \& Rabbitt, 1989; Posner, 1980). This effect could be stimulus driven (Theeuwes, 1992) or goal dependent (because participants could have searched for the singleton to find the target; see Bacon \& Egeth, 1994). In addition, if the prime singleton activated a (left vs. right) response corresponding to its location (see Ansorge \& Neumann, 2005; Leuthold \& Kopp, 1998; Neumann \& Klotz, 1994), the prime singleton should also facilitate responding to the target singleton appearing at the same location (and, therefore, requiring the same response). In contrast, in the incongruent condition, the prime singleton appeared on the side opposite the singleton target. Thus, the incongruent prime could interfere with the response to the target singleton both by attentional capture away from the target singleton and by activating the alternative response to that required by the target. 
Prime

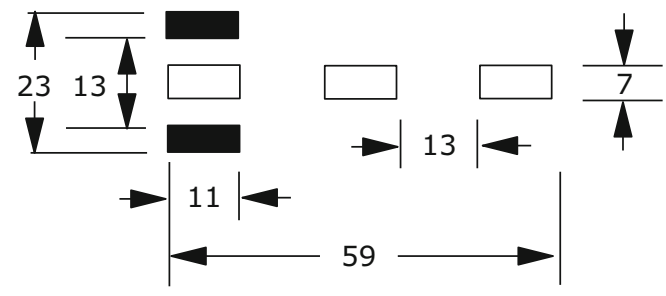

Target

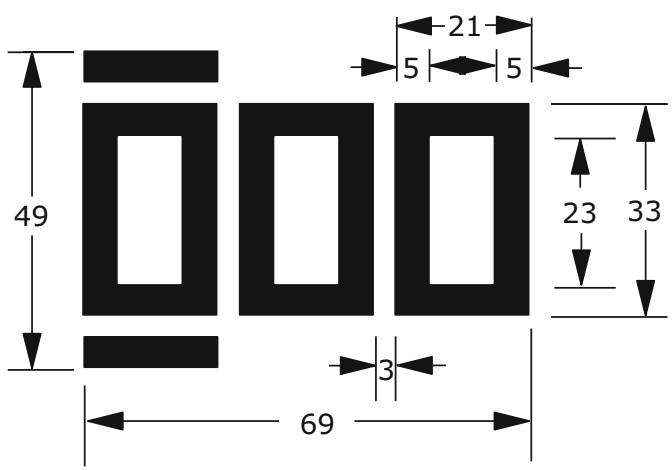

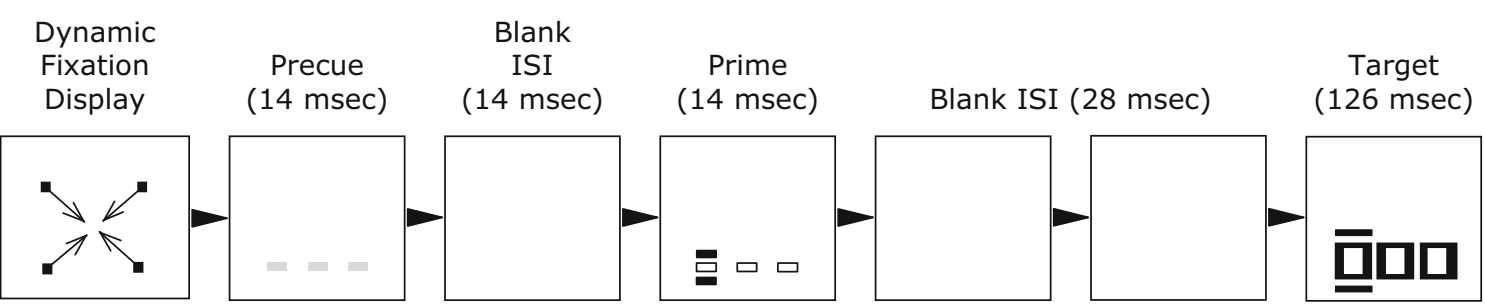

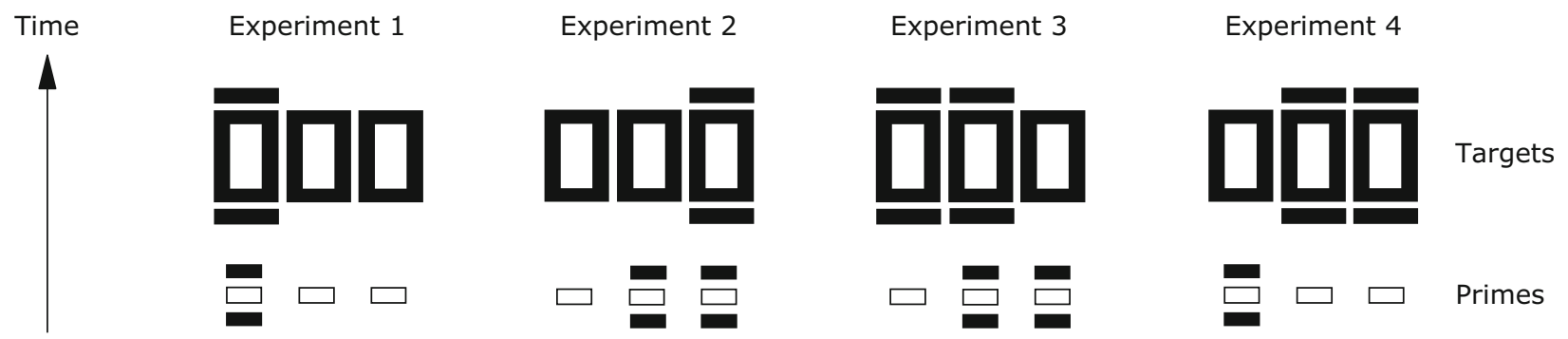

Figure 1. Upper panel: Examples of a prime display (on the left) and of a target display (on the right), both with sizes in pixels. Arrows indicate boundaries for the size measurements. Middle panel: Sequence of events on a trial from left to right. Depicted is a congruent trial in Experiment 1, with the target on the left. Primes were presented for $\mathbf{2 8}$ msec on half of the trials. Arrows depict the flow of time. Lower panel: Examples of prime-target combinations in the four different experiments (left to right: 1-4): left, an example of a congruent trial in Experiment 1; second from left, an example of a feature-congruent/singleton-incongruent trial in Experiment 2; second from right, an example of an incongruent feature-absent trial in Experiment 3; right, an example of a feature-incongruent/ singleton-congruent trial in Experiment 4. The stimuli are not drawn to scale. Arrows depict the flow of time. For further information, refer to the text. ISI, interstimulus interval.

In Experiments 1-4, we did not attempt to dissociate between these two conceivable effects, because we wanted to use a maximally encompassing measure for our test, one capable of registering any of several potential effectsstimulus driven, goal directed, attentional capture, and/or motor activation - of the masked singleton. Investigation of the origin of the singleton effects was postponed to the final Experiment 5.

We also used a second task to verify low prime bar visibility. Prime visibility was tested in separate blocks after the CRT. In the prime visibility test blocks, participants had to discriminate between a singleton prime on the left and one on the right. This task required discrimination of the same prime singleton feature (its location) that was also responsible for its expected congruence effect in the CRT. This prime visibility test is therefore a fair measure for computation of a correlation with the priming effect in the CRT.

Our expectations concerning the visibility test were as follows. If the singleton prime's congruence effect crucially depended on the participants' awareness of the singleton, we should obtain a significant correlation between singleton prime visibility and the congruence effect in the CRT (Holender \& Duscherer, 2004). However, if the congruence effect in the CRT did not depend on participants' awareness of the singleton prime, as we expected (see Zhaoping, 2008), the correlation between the singleton prime's congruence effect in the target CRT and the 
singleton prime's visibility should be low and nonsignificant (see Ansorge, 2003; Greenwald, Draine, \& Abrams, 1996; Naccache \& Dehaene, 2001). Only if we found a low correlation would it be possible to conclude that our procedure worked as planned and that we indeed measured processing of prime singletons remaining outside of participants' awareness.

In addition, we tested whether the masked primes were presented below an objective threshold of prime visibility; that is, visibility scores were tested for significant differences from chance performance (cf. Snodgrass, Bernat, $\&$ Shevrin, 2004). Processing of masked primes below the objective threshold has only rarely been demonstrated (see Holender, 1986; Reingold, 2004; Reingold \& Merikle, 1988; T. Schmidt \& Vorberg, 2006), although it has been sometimes found with the procedure and stimuli used in the present experiment (Ansorge, 2003; Ansorge \& Neumann, 2005; Neumann \& Klotz, 1994). However, in comparison to prior studies, we used a slightly different and very sensitive procedure to assess prime visibility (for details, refer to the General Discussion). Therefore, we did not necessarily expect that our visibility measure also demonstrated processing of masked primes below an objective threshold of awareness.

\section{Method}

Participants. Eleven participants ( 6 of them female), including the first author, participated in Experiments 1-4; they all performed the experiments in the same order. The participants were between 19 and 30 years of age, had normal or corrected-to-normal vision, and were paid for their participation (except for the first author).

Stimuli and Procedure. The stimuli were presented black on white on a computer cathode ray tube monitor with a refresh rate of $71 \mathrm{~Hz}$ (i.e., 14-msec frame duration); each pixel covered an area of $0.32 \times 0.32 \mathrm{~mm}$, corresponding to roughly $0.03^{\circ} \times 0.03^{\circ}$ of visual angle at a viewing distance of $60 \mathrm{~cm}$.

See also Figure 1 for the exact sizes of all stimuli (in the upper row), an example of the sequence of events in a trial (in the middle row), and an illustration of the major manipulations in the first four experiments. Each trial started with a blank screen for $700 \mathrm{msec}$. Next, a dynamic fixation display was presented, accompanied by a brief alerting tone $(700 \mathrm{~Hz})$. The fixation display consisted of four dots (made up of 4 pixels each) that appeared on the two diagonal screen axes at an eccentricity of $4.5^{\circ}$ and then moved, over an 800 -msec zooming-in period, toward the screen center, where they merged and vanished. The next frame consisted of three small precues: one central, one left, and one right of the screen center. These precues were dotted rectangular patterns $\left(1.8^{\circ}\right.$ wide $\times 0.76^{\circ}$ high; luminance $=62 \mathrm{~cd} / \mathrm{m}^{2}$ ) presented for $14 \mathrm{msec}, 1.53^{\circ}$ above or below fixation. Next, there was one blank screen for $14 \mathrm{msec}$, which was immediately followed by three outline prime boxes (11 pixels wide $\times 7$ pixels high) at the former positions of the precues. One of the lateral prime boxes, the prime singleton, had two black flanking prime bars ( 11 pixels wide $\times 7$ pixels high), one above and the other below the priming box. The primes were presented for 14 or $28 \mathrm{msec}$ (with duration varying between blocks). After prime offset, the screen remained blank for two frames $(28 \mathrm{msec})$, and then the target was shown. Accordingly, stimulus onset asynchronies (SOAs) between the prime and target depended on prime duration: The SOA was $42 \mathrm{msec}$ with the short primes and $56 \mathrm{msec}$ with the long primes. The target display consisted of three larger outline boxes (21 pixels wide $\times 31$ pixels high; line thickness, 5 pixels) that surrounded the prime boxes plus the prime bars. These were shown for $126 \mathrm{msec}$. Because of the short SOA between the prime display and target dis- play, the prime boxes and prime bars were metacontrast-masked by the surrounding larger target display boxes (cf. Neumann \& Klotz, 1994). As with the primes, only one of the lateral target boxes had two flanking bars (each 21 pixels wide $\times 5$ pixels high), one bar above and the other below the box. This was the singleton target for the CRT. On congruent trials, the prime bars and target bars were presented at the same position (e.g., both were right); on incongruent trials, they appeared on opposite sides (e.g., prime bars left, target bars right). In the prime visibility test (see below), an additional answer display was shown following the target display: a $15.5 \times 20 \mathrm{~cm}$ rectangular area that consisted of four fields of equal size specifying four possible answers - namely, "certain left," "left," "right," and "certain right," referring to the side of the prime. The answer display was presented until the participants made the required judgment.

The participants had two tasks. In the target CRT, the participants responded as quickly and accurately as possible to the side of the visible singleton target. If the visible singleton target was on the left, the participants had to press a left key; if it was on the right, the participants had to press a right key. In the prime visibility test, the participants had to judge on which side the masked singleton prime had been shown. It was carefully explained to the participants what the masked primes looked like. Judgments were given by selecting the answer square on the display via moving the mouse cursor into that respective field and clicking the mouse. The four answer fields were displayed at trialwise randomized positions, so as to ensure that the judgments truly reflected prime visibility and to preclude that they could be given on the basis of capture by or response activation effects of the masked singleton primes (even though these primes may have been outside of awareness).

The two tasks were administered in successive blocks, with target CRT before prime visibility test; there were two pairs of such (target CRT plus prime visibility test) blocks, one for each of the two prime-duration/SOA conditions. The sequence of blocks ensured that performance in the visibility test benefited from dark adaptation (see Holender, 1986) and learning (Wolford, Marchak, \& Hughes, 1988), meaning that the visibility test's sensitivity was maximized, and the procedure had the additional advantage that the participants could fully concentrate on each task in turn. Each block consisted of 70 repetitions of 2 prime positions (left, right) $\times 2$ target positions (left, right). Left and right positions of the prime and target were equally frequent and varied orthogonally. Prior to the visibility test, the participants were shown slow-motion versions of the trial events, and it was carefully explained to them that the primes were much harder to see and might take on a different, "seen" form if masked. Together with instructions and practice, the experiment took about $70 \mathrm{~min}$ to complete.

\section{Results}

Target CRT. Significance was assumed at a $p<.05$. See Figures 2 and 3 for the results. A Holm test of the correct reaction times (RTs) revealed that RTs were significantly shorter on congruent trials at both short and long SOAs, as compared with incongruent trials at short and long SOAs [short SOAs, 299 vs. $347 \mathrm{msec}$; long SOAs, 299 vs. $369 \mathrm{msec} ; t \mathrm{~s}(10)=7.93$ and 12.68, respectively]. Furthermore, the congruence effect (incongruent RT congruent RT) was significantly larger at the long than at the short SOA $[t(10)=4.03]$.

A Holm test of the arc-sine transformed percentages of errors (PEs) corroborated the findings. The mean PEs were significantly lower on congruent trials at short and long SOAs, as compared with incongruent trials [short SOAs, $0.58 \%$ vs. $8.38 \%$; long SOAs, $0.39 \%$ vs. $12.08 \%$; ts $(10)=$ 5.42 and 13.02 , respectively]. Once again, the congruence effect was larger at the long SOA $[t(10)=3.43]$. 


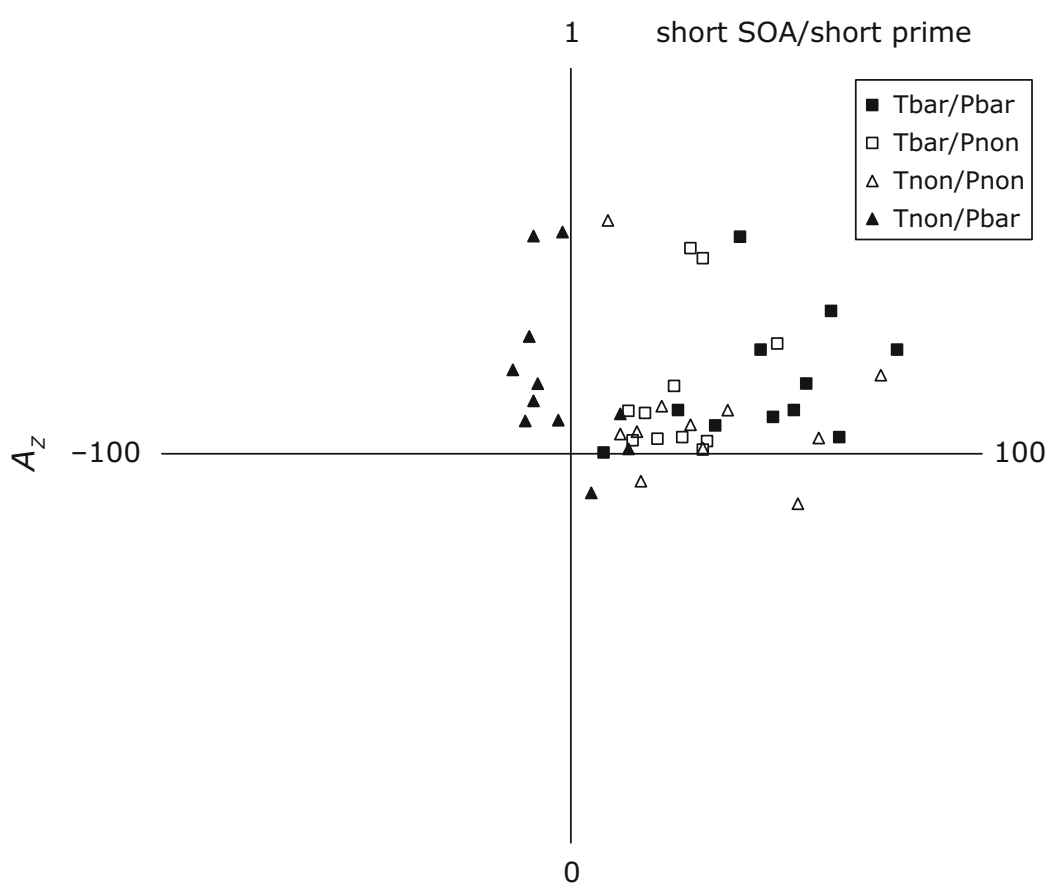

RT Congruence (msec)

Figure 2. Response time (RT) congruence effects in milliseconds (incongruent RT - congruent RT) for the target choice response task on the $x$-axis as a function of prime visibility (expressed as $A z$ ) on the $y$-axis in the short-prime/short-SOA condition. Each data point corresponds to the performance of one participant. Black squares (Tbar/Pbar) represent data from Experiment 1, in which singleton targets (Tbars) and singleton primes (Pbar) consisted of bars. White squares (Tbar/Pnon) represent data from Experiment 2, in which singleton targets consisted of bars (Tbars) and singleton primes (Pnon) consisted of blank squares without bars. White triangles (Tnon/Pnon) represent data from Experiment 3, in which singleton targets (Tnon) and singleton primes (Pnon) consisted of blank squares without bars. Black triangles (Tnon/Pbar) represent data from Experiment 4, in which singleton targets consisted of blank squares without bars (Tnon) and singleton primes consisted of bars (Pbar). Note that individual visibility scores were very low and that, across participants, correlations between visibility scores and RT congruence effects were low. SOA, stimulus onset asynchrony.

Prime visibility. Subjectively, as assessed by verbal interview after the CRT and prior to the visibility test, none of the participants had seen the masked primes. Objectively, prime visibility was assessed by methods of signal detection theory (SDT; see Green \& Swets, 1966), which provide very sensitive measures of residual awareness (see Klotz \& Neumann, 1999; Reingold \& Merikle, 1988). We determined individual hit rates as the percentages of judgments affirming that the prime singleton was certainly on the right when the prime singleton was, in fact, on the right, and false alarm (FA) rates as the percentages of judgments affirming that the prime singleton was certainly on the right when the prime singleton was, in fact, on the left (and not on the right) — and proceeding analogously for the three remaining judgments ("right," "left," and "certainly left"). Next, we assessed the participants' individual sensitivity for correctly reporting the prime singleton. To that end, we computed the receiver operating characteristic (ROC) curve. For each certainty level, hit rates on one coordinate were plotted against FA rates on a second coordinate. The line connecting the resulting points gives the ROC curve. As an index of the prime's visibility, we computed a value corresponding to the area under the ROC curve, $A_{z}$, using Dorfman's (1982) RSCORE II program (see also Dorfman \& Alf, 1969). Informally, $A_{z}$ values can be readily interpreted if considered as proportions of correct judgments, with a value of .50 denoting discrimination at chance level and an $A_{z}$ value of 1.0 denoting perfect discrimination performance.

$A_{z}$ values were tested by Holm tests. The discriminability of the left and right primes in the short-SOA condition (mean $A_{z}=.593$ ) was low but significantly above chance $[t(10)=2.91]$. In the long-SOA condition, the primes were also discriminated with better-than-chance accuracy (mean $\left.A_{z}=.671\right)[t(10)=5.34]$ and were discriminated better than in primes in the short-SOA condition $[t(10)=$ 4.04]. Across participants, the correlation between individual RT congruence effects and $A_{z}$ values was low and nonsignificant at both long $[r(11)=.168, p=.604]$ and short $[r(11)=.229, p=.476]$ SOAs. Intercepts of lin- 


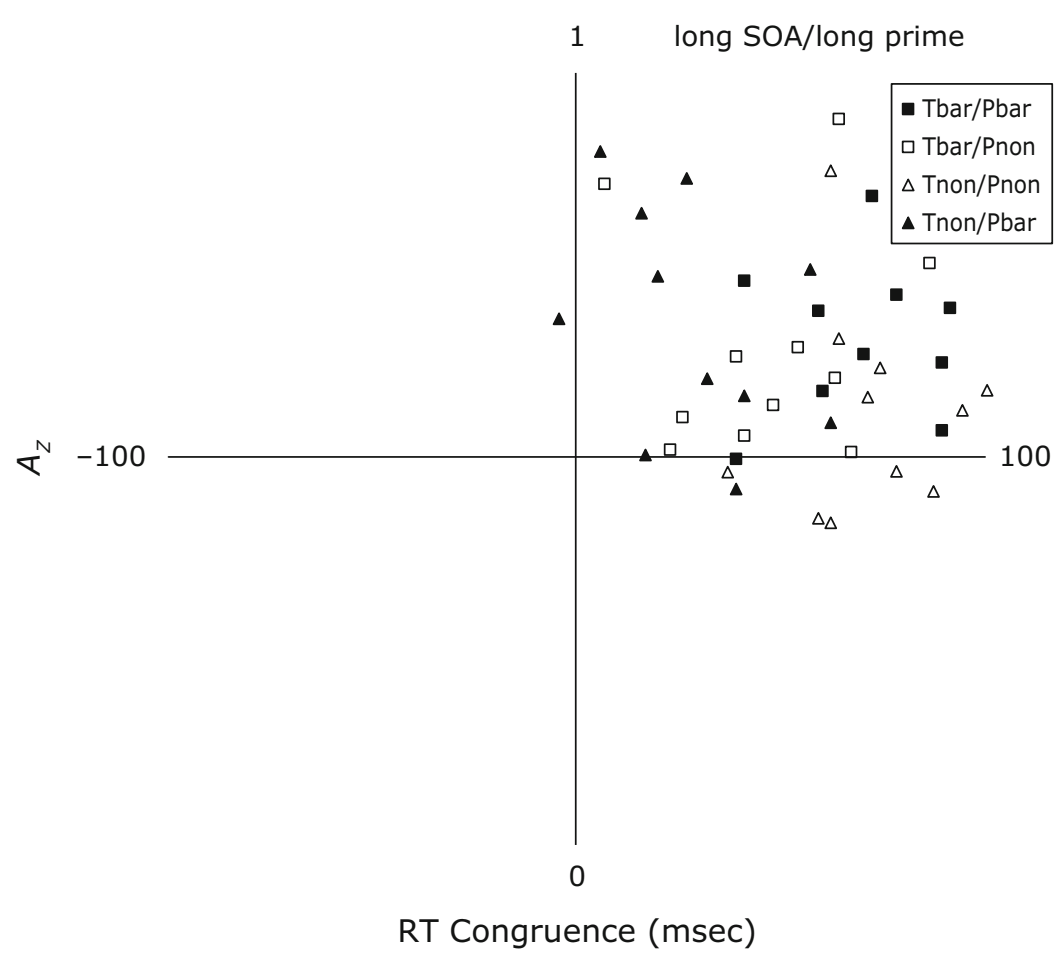

Figure 3. Same as Figure 2, but for long-prime/long-SOA conditions. SOA, stimulus onset asynchrony.

ear regressions that regressed individual RT congruence effects (incongruent RT - congruent RT) on individual prime visibility scores $\left(A_{z}\right)$ to assess congruence effects at zero awareness (see Greenwald et al., 1996) were nonsignificant at both short and long SOAs $(t \mathrm{~s}<1)$.

\section{Discussion}

We found that the masked prime singletons produced a significant congruence effect in the CRT. The congruence effect was not significantly correlated with the prime singleton's visibility. Together with the participants' subjective impression that the prime was not seen, the results suggest that masked primes exert an effect even if presented below a subjective level of awareness. In contrast to previous studies (e.g., Neumann \& Klotz, 1994), our participants discriminated the primes with above-chance accuracy. Therefore, dissociations between the congruence effect in the CRT task and the prime visibility were based only on a lack of a correlation and must be interpreted cautiously, (1) by assuming that the dissociation was "only" quantitative, rather than qualitative, in nature; (2) by bearing in mind that an insignificant correlation can never "prove" that perception and priming were fully dissociated; and (3) by assuming that if the visibility scores are of no significant predictive value for the congruence effect, the congruence effect is not a visibility-dependent effect (Holender \& Duscherer, 2004).

In the present experiment, the masked prime was a singleton. Thus, the prime's congruence effect in the CRT could have reflected singleton capture or singletonelicited motor activation. Therefore, the data appear to be in line with the conclusions of Zhaoping (2008). However, the present masked congruence effect could also be explained by feature search (see Folk, Remington, \& Johnston, 1992; Leber \& Egeth, 2006). To solve the CRT, the participants could have searched for the defining features of the target-namely, its bars-altogether ignoring the singleton status of the target and the prime. On this view, with the participants set to search for a pair of target bars, the similar bars possessed by the masked prime could have attracted attention. If this were true, the observed congruence effect would not be due to singleton capture. In line with this, prior research has shown that a masked prime possessing a searched-for feature attracted attention even if this prime was a nonsingleton (see Ansorge, Kiss, \& Eimer, 2009). Experiment 2 was designed to disentangle the potential influences of prime features and prime singletons on the congruence effect.

\section{EXPERIMENT 2}

In Experiment 2, the influences of the prime's singleton status were pitted against the influences of the prime's feature status. The participants again searched for one singleton target with flanking bars. In contrast to Experiment 1, however, the prime that preceded the target now consisted of two pairs of bars, one lateral (left or right), and the other one central on the screen (see Figure 1). As before, the lateral bars in the prime display were either congruent or incongruent with the target position. This kind of congruence was termed feature-congruence. Under the present double-bar-prime conditions, the singleton prime was 
the one priming box without flanking bars. This "blank" prime or singleton prime was at the side opposite the feature prime. Thus, feature-congruent conditions were also singleton-incongruent conditions, and feature-incongruent conditions were singleton-congruent conditions.

The expectations for Experiment 2 were as follows. If the feature prime exerted a congruence effect, RTs in the CRT were expected to be lower in feature-congruent/singletonincongruent conditions than in feature-incongruent/ singleton-congruent conditions. In contrast, if the singleton prime captured attention or activated a response, RTs were expected to be lower in feature-incongruent/ singleton-congruent conditions than in feature-congruent/ singleton-incongruent conditions. A mixture of singletonand feature-based effects should lead to a diminished congruence effect, as compared with Experiment 1, because in Experiment 1, both singleton-based and feature-based effects pulled in the same direction.

\section{Method}

In the CRT, the only change, as compared with Experiment 1, was that the central prime box was additionally marked by flanking bars (see also Figure 1). In the prime visibility test, the participants again had to report the location of the prime singleton. It was carefully explained that the singleton was the prime box without bars. No participant reported problems understanding the visibility task.

\section{Results}

Target CRT. See Figures 2 and 3 for the results. Holm tests of the correct RTs revealed that responses on featurecongruent/singleton-incongruent trials at short and long SOAs were significantly faster than responses on featureincongruent/singleton-congruent trials [short SOAs, 296 vs. $323 \mathrm{msec}$; long SOAs, 297 vs. $344 \mathrm{msec} ; \mathrm{ts}(10)=8.75$ and 6.82, respectively]. As in Experiment 1, this congruence effect was stronger at the long SOA $[t(10)=3.39]$.

For the PEs, Holm tests corroborated these findings. The mean PEs on feature-congruent/singleton-incongruent trials at short and long SOAs were significantly lower than those on feature-incongruent/singleton-congruent trials [short SOAs, $1.36 \%$ vs. $6.17 \%$; long SOAs, $1.49 \%$ vs. $10.45 \% ; t \mathrm{~s}(10)=7.14$ and 6.28 , respectively]. This feature congruence effect was stronger at the long than at the short SOA $[t(10)=2.88]$. The mean PEs and RTs on feature-congruent trials were virtually the same at both SOAs: As in Experiment 1, altering the prime duration affected the speed and accuracy of responses only on feature-incongruent trials.

The congruence effect in target CRTs mostly reflected feature priming, rather than singleton priming. However, the opposing singleton-priming and feature-priming effects in Experiment 2 canceled each other out, at least in part-as is attested by the significantly reduced net congruence effect (incongruent RT - congruent RT) with both short SOAs and long SOAs, in comparison with Experiment 1 [short SOA, 27 vs. $48 \mathrm{msec}$; long SOA, 47 vs. $70 \mathrm{msec}$ in Experiments 2 and 1, respectively; ts $(10)=$ 4.34 and 3.02, respectively].

Prime visibility. Holm tests of the mean $A_{z}$ values revealed discrimination performance to be significantly above chance only with long SOAs $\left(A_{z}=.624\right)$, but not with short SOAs $\left(\mathrm{A}_{z}=.537\right)[t \mathrm{~s}(10)=2.43$ and 1.12 , respectively]. In addition, discrimination accuracy differed significantly between the two SOA conditions $[t(10)=$ 2.39]. The correlation between individual RT congruence effects and $A_{z}$ scores showed no systematic and significant relationship between the two variables, for either short $[r(11)=.369, p=.264]$ or long $[r(11)=.098, p=$ $.774]$ SOAs. Intercepts of linear regressions that regressed individual RT congruence effects (incongruent RT - congruent RT) on individual prime visibility scores $\left(A_{z}\right)$ were significant at both short $[t(10)=2.74, p<.05]$ and long $[t(10)=2.74, p<.05]$ SOAs, demonstrating congruence effects at zero awareness (see Greenwald et al., 1996).

\section{Discussion}

Experiment 2 confirmed the significant congruence effect in target CRTs, in the absence of a significant correlation between RT congruence effect and singleton prime visibility. The congruence effect in target CRTs reflected mostly feature priming. Yet, as evidenced by the significantly reduced net congruence effects (with short and long SOAs) in Experiment 2, relative to Experiment 1 , the singleton-priming effect partially canceled the feature-priming effect. It is also conceivable that a feature's singleton status boosted a feature-priming effect in Experiment 1, but not in Experiment 2 (e.g., Lamy $\&$ Zoaris, 2009). In any case, we confirmed Zhaoping's (2008) assumption that singletons outside participants' awareness can be processed.

We also found a slight difference in the visibility data, as compared with Experiment 1: Singleton prime visibility was lower and, in fact, not significantly different from chance in the short-SOA condition in Experiment 2, whereas it was significantly above chance in this condition in Experiment 1. This would leave open the possibility that the participants misunderstood the task of reporting the singleton and, instead, relied on the singleton target for their report. Note, however, that none of the participants reported problems understanding the task (of reporting the presence of the masked blank boxes without the bars), making it unlikely that the reduced visibility scores in Experiment 2 are attributable to misunderstandings of the task. The results may thus be taken to indicate that it is more difficult to locate a feature-absent than a feature-present singleton prime. This makes sense in light of the well-known increase in search difficulty for featureabsent stimuli in visual search (Treisman \& Gormican, 1988; Treisman \& Souther, 1985). The results also suggest that, at least with the short SOA, the primes were presented below an objective threshold of awareness (see Neumann \& Klotz, 1994).

Yet caution is advised. It is possible that part of the participants' ability to discriminate the primes had little to do with the prime's visibility, and more with the changed perception of the target displays (Reingold \& Merikle, 1988). Visual motion features created by the succession of prime and mask are known to survive metacontrast masking (see Ansorge, Breitmeyer, \& Becker, 2007; Kolers, 1963). 
Thus, perceived expansion created by less eccentric local prime bars being succeeded by more eccentric local target bars could have provided a cue for judging the position of the prime in Experiment 1, but not in Experiment 2.

In addition, with sufficient power, probably any visibility measure can pass the objective threshold. Here, it suffices to note that prime visibility was definitely less than suggested by the substantial priming effects obtained in the CRT, and that low and nonsignificant correlations between visibility scores and congruence effects during the CRT indicated that residual awareness of the primes is not the main source of the congruence effect.

\section{EXPERIMENT 3}

Experiment 2 suggested that even a masked featureabsent singleton was processed. This is important. According to computational theories, feature differences between neighboring stimuli, rather than the stimulus energy of a particular stimulus, account for singleton effects. The feature-absent prime in Experiment 2 was a singleton defined by a feature difference from its neighbors, although it was of slightly lower energy than the nonsingletons since it lacked the bars possessed by neighboring stimuli. Thus, Experiment 2 was in line with a crucial prediction deriving from computational singleton-contrast accounts (see Itti \& Koch, 2001). On the other hand, the prime's congruence effect with a feature-present singleton prime in Experiment 1 exceeded that of a feature-absent singleton prime in Experiment 2, which is more in line with Zhaoping's $(2005,2008)$ assumption that V1 feature processing underlies salience-driven effects.

Experiment 3 was designed to further investigate this issue. To assess the maximal congruence effect of featureabsent singleton primes, we repeated the conditions in Experiment 1 , with, however, the roles of bar-flanked boxes and non-bar-flanked boxes reversed: That is, in Experiment 3 , a visible box without flanking bars was used as the singleton target and as a masked singleton prime. To find the visible target, bar-flanked boxes at the central position and the position opposite the target had to be disregarded. If singleton prime boxes without flanking bars suffer from less energy, one would expect a diminished congruence effect of the feature-absent primes in Experiment 3, as compared with the effect of feature-present primes in Experiment 1 . However, if singleton primes exert their effect via feature differences from the neighboring stimuli, one might expect equivalent congruence effects by the masked feature-absent (Experiment 2) and feature-present (Experiment 1) primes.

Visual search is easier for feature-present than for feature-absent stimuli (see Treisman \& Gormican, 1988; Treisman \& Souther, 1985). In Experiment 3, the singleton target was defined by feature absence: It was the one box without bars (see also Figure 1). Consequently, we might also find that it takes, overall, longer to respond to the targets in Experiment 3, as compared with those in Experiment 1 (in the latter, targets were defined by feature presence).

\section{Method}

The two changes were compared with Experiment 1 (see also Figure 1): The central target and prime boxes were flanked by bars, and the participants now had to search for and respond to the side of the feature-absent target.

\section{Results}

CRT task. See Figures 2 and 3 for the results. A Holm test of the correct RTs in Experiment 3 revealed responses to be significantly faster at short and long SOAs in the congruent condition, as compared with the incongruent condition [short SOA, 311 vs. $345 \mathrm{msec}$; long SOA, 315 vs. $387 \mathrm{msec}$, for congruent and incongruent conditions, respectively; $t \mathrm{~s}(10)=5.09$ and 13.14 , respectively]. Again, the congruence effect was stronger at long than at short SOAs $[t(10)=4.82]$.

Mean PEs were also significantly lower on congruent trials at short SOAs and long SOAs, as compared with incongruent trials [short SOA, $1.23 \%$ vs. $9.87 \%$; long SOA, $0.39 \%$ vs. $14.61 \%$; ts $(10)=9.4$ and 10.91 , respectively]. Again, the difference between congruent and incongruent trials was larger at long than at short SOAs $[t(10)=$ 2.25], and the stronger congruence effect for long SOAs was owing mostly to incongruent trials.

To investigate the difference between feature-present and feature-absent priming effects, mean correct RTs and PEs were also compared between Experiments 1 and 3 in two separate three-way ANOVAs, both with the factors of experiment $(1,3)$, prime duration (short, long), and congruence (congruent, incongruent). Mean RTs were significantly lower in Experiment 1 than in Experiment 3 $[329$ vs. 340 msec; $F(1,10)=13.29, p<.01]$. Mean PEs tended to be lower in Experiment 1 than in Experiment 3 $[5.36 \%$ vs. $6.53 \% ; F(1,10)=4.41, p=.062]$. Thus, responses were indeed faster and more accurate to featurepresent targets (Experiment 1) than to feature-absent targets (Experiment 3 ). In addition, there was a marginally significant experiment $\times$ congruence interaction in the RT data $[F(1,10)=4.27, p=.066]$, reflecting a tendency for the congruence effect to be reduced for the featureabsent singleton primes in Experiment 3 (see also below) as compared with the feature-present primes in Experiment 1 . None of the remaining interactions involving the two factors of experiment and congruence were significant (all $p \mathrm{~s}>.10$ ), either for RTs or for PEs.

On closer inspection (i.e., pairwise comparisons of congruence effects between experiments, separately for short and long SOAs), only the congruence effect at short SOAs was found to decrease from feature-present (Experiment 1) to feature-absent (Experiment 3) prime conditions [ 48 vs. $33 \mathrm{msec} ; t(10)=2.53$ ]. The congruence effect at long SOAs was equivalent for feature-present and feature-absent primes [70 and $72 \mathrm{msec}$, respectively; $t(10)=0.37]$.

Prime visibility. Mean $A_{z}$ values were not significantly above chance in blocks with short $\left(A_{z}=.552\right)$ and long $\left(A_{z}=.559\right)$ SOAs $[t \mathrm{~s}(10)=1.78$ and 1.47 , respectively $]$ and were equivalent for the two SOAs $[t(10)=0.37]$. Individual RT congruence effects and $A_{z}$ values were not significantly correlated across participants, either at short 
SOAs $[r(11)=-.240, p=.478]$ or at long SOAs $[r=$ $.042, p=.903]$. In line with congruence effects at zero prime awareness, intercepts of linear regressions that regressed individual RT congruence effects (incongruent RT - congruent RT) on individual prime visibility scores $\left(A_{z}\right)$ approached significance at short SOAs $[t(10)=2.15$, $p=.06]$ and were significant at long SOAs $[t(10)=4.31$, $p<.01]$.

\section{Discussion}

The feature-absent primes in Experiment 3 led to a significant congruence effect. In addition, for short SOAs, this effect was diminished, relative to the feature-present primes in Experiment 1. This suggests that feature energy plays a role for the congruence effect produced by masked singleton primes. However, the strong congruence effect at long SOAs supports a clear-cut interpretation in terms of a singleton effect: A singleton prime's effect should depend on a feature difference between adjacent prime boxes; prime energy as such should matter less. The fact that, at long SOAs, the congruence effect elicited by featureabsent singleton primes (Experiment 3 ) was as large as that elicited by feature-present primes (Experiment 1) indicates that local feature differences gave rise to the congruence effect, as predicted by contrast-driven singleton effects (see Itti \& Koch, 2001); by contrast, the results with short SOAs again pointed toward a role for feature singleton effects ( $\mathrm{Li}, 2002)$. Taken together, these findings suggest that the time courses of contrast-driven and feature-driven awareness-independent priming effects might differ.

Again, the correlation between prime visibility and the magnitude of the RT congruence effect was low and nonsignificant. This finding suggests that the congruence effect did not depend on the participants' awareness of the prime singleton. Again, on average, prime discrimination performance did not differ significantly from chance. As in Experiment 2, this result might indicate that primes were presented below an objective threshold of awareness (although, as with Experiment 2, we prefer to interpret this with caution).

In addition, searching for feature-absent targets was indeed more difficult than searching for feature-present targets (see Treisman \& Gormican, 1988): RTs and PEs were significantly increased for feature-absent targets (Experiment 3), as compared with feature-present targets (Experiment 1). This is in contrast to Itti and Koch's (2001) contrast-driven singleton capture explanation but fits nicely with Li's (2002) assumption that local feature contrasts defined by feature presence lead to direct V1 activation and, thus, higher amplitudes, as compared with singletons or local contrasts defined by the absence of a feature.

\section{EXPERIMENT 4}

Experiment 4 was designed to replicate Experiment 2, but with feature-absent targets; that is, the singleton target was the one box without flanking bars, presented to the left or right of two adjacent boxes, both of which were flanked by bars. There was also one singleton bar prime shown to the left or right; the other two prime boxes were without flanking bars (see Figure 1, lower panel). Under these conditions, congruence effects could have had two origins. First, they could have been based on the position of a prime with a target-matching "feature" if it is assumed that participants can search for a target with an absent feature (here, the absent bars) much as they can search for the present features. This would lead to capture by the featurematching prime - here, a priming box without bars. (Although this prime was, in fact, a feature-absent prime [as in Experiment 2], for the sake of consistency, we refer to the congruence relation based on this target-similar prime as feature congruence.) Second, congruence effects could have been based on the position of the prime singleton. Consistent with Experiment 2, we refer to this type of congruence as singleton congruence. As in Experiment 2, feature-congruent conditions were also singleton incongruent, and feature-incongruent conditions were singleton congruent. If singleton primes were processed, we expected the feature-congruence effect in Experiment 4 to be lower than that in Experiment 3, in which singleton priming and feature priming worked in unison and added up to the overall congruence effect.

\section{Method}

As compared with Experiment 1, the changes were that the central target box was additionally flanked by bars and that the participants now had to respond to the side of the singleton target defined by feature absence (see also Figure 1).

\section{Results}

CRT task. See also Figures 2 and 3 for the results. For the correct RTs, a Holm test showed that at long SOAs, RTs were significantly shorter on feature-congruent/singletonincongruent trials than on feature-incongruent/singletoncongruent trials [327 vs. $356 \mathrm{msec} ; t(10)=4.65$ ]. At short SOAs, RTs did not differ between the feature-congruent/ singleton-incongruent and feature-incongruent/singletoncongruent conditions [324 vs. $321 \mathrm{msec} ; t(10)=1.17$, $p=.269]$. Increasing the SOA increased the feature congruence effect $[t(10)=4.96]$.

The Holm test of the PEs partly supported these results. At long SOAs, significantly fewer errors were made on feature-congruent/singleton-incongruent trials than on feature-incongruent/singleton-congruent trials [3.57\% vs. $8.96 \% ; t(10)=5.06]$. In contrast, at short SOAs, the PEs did not differ between feature-congruent/singletonincongruent trials and feature-incongruent/singletoncongruent trials $[4.74 \%$ vs. $4.09 \% ; t(10)=0.66, p=.52]$. Increasing the SOA increased the feature congruence effect in the PEs $[t(10)=4.56]$, and also changed its sign.

Singleton primes diminished the congruence effect in Experiment 4, where they worked in the direction opposite to the feature-priming effect, relative to Experiment 3, where they worked in unison with the feature-priming effect. This decrease was found at both SOAs: At long SOAs, the congruence effect dropped from $72 \mathrm{msec}$ in Experiment 3 to $29 \mathrm{msec}$ in Experiment 4; at short SOAs, it dropped from 33 to $-3 \mathrm{msec}[t \mathrm{~s}(11)=4.74$ and 4.43 , respectively]. 
Prime visibility. The Holm test of the $A_{z}$ values revealed accuracy to be significantly above chance at both long $\left(A_{z}=.677\right)$ and short $\left(A_{z}=.601\right)$ SOAs $[t \mathrm{~s}(10)=$ 3.96 and 3.15, respectively], with overall higher accuracy at long SOAs $[t(10)=2.29]$. However, the congruence effect was not significantly correlated with the $A_{z}$ values, either at long SOAs $[r(11)=.395, p=.207]$ or at short SOAs $[r(11)=.405, p=.195]$. Intercepts of linear regressions that regressed individual RT congruence effects (incongruent RT - congruent RT) on individual prime visibility scores $\left(A_{z}\right)$ were nonsignificant at short SOAs $[t(10)=1.16, p=.27]$ but significant at long SOAs $[t(10)=2.23, p=.05]$.

\section{Discussion}

As in Experiment 2, pitting "feature priming" (by a target-similar prime box) against singleton priming, we found a dominance of "feature priming" in determining the congruence effect. But, as was the case in Experiment 2, the singleton primes were also processed: They diminished the congruence effect in Experiment 4, in which they worked in the direction opposite to the featurepriming effect. This led to a decreased congruence effect, relative to Experiment 3, in which singleton priming worked in unison with the feature-priming effect. This diminishing effect of singleton priming was stronger in Experiment 4 than in Experiment 2. This could be due to the additional attention-capturing potential of the higher energy of the singleton primes with bars that we used in Experiment 4, in comparison with the singleton primes without bars used in Experiment 2.

In addition, a low correlation between visibility and congruence effect again indicated that prime visibility was unlikely to be responsible for the congruence effect. This conclusion was backed up by subjective reports: None of the participants reported having seen the primes in any of the experiments. Together, the results confirmed that singletons remaining outside participants' awareness are nevertheless processed.

\section{EXPERIMENT 5}

Experiment 5 had three aims. The first was to test whether masked singletons can capture attention. This became necessary because Experiments 1-4 did not permit us to dissociate the attention-capturing effect of a masked prime singleton from its response-activating effect. To allow for this, in the CRT in Experiment 5, response activation by the masked prime singletons was prevented. Participants were shown shape-heterogeneous target displays and had to respond to the (angular) outline shape of the visible target (i.e., they had to indicate whether the target was a diamond or a square), while a small ring was used as a masked prime. Importantly, rather than being shape defined, the singleton prime differed in color from three concomitant color-homogeneous rings (see Figure 4). Thus, because the color singleton prime was very different in shape from the response-relevant target forms, it could not activate one or the other of the responses associated with the targets.
By contrast, attentional capture by the masked singleton prime was possible. In Experiment 5A, the visible target was defined as the one color singleton per trial, with singleton color changing unpredictably from trial to trial and participants having to respond to the shape of this singleton. Under these conditions, the participants were not able to search for a particular color but had to look for a color-defined singleton to detect the target (see Bacon \& Egeth, 1994). Given this, a masked color singleton prime was expected to capture attention, reflected in enhanced performance when the masked color singleton prime preceded the color singleton target at the same, rather than at a different position. This prediction holds at least for prime-target intervals shorter than $150 \mathrm{msec}$ (see Kim \& Cave, 1999; see also below).

The second aim of Experiment 5 was to examine whether masked color singleton primes would capture attention in a stimulus-driven or a top-down-controlled manner. To this end, the masked-priming effects in Experiment $5 \mathrm{~A}$ (color singleton prime $\rightarrow$ color singleton target) were compared to those in Experiment 5B, in which participants searched for a visible shape singleton target which was, however, again preceded by a masked color singleton prime (color singleton prime $\rightarrow$ shape singleton target). If the masked color singleton prime captured attention in a stimulus-driven manner, a masked-priming effect would be expected in Experiment 5B, as well as in Experiment 5A (see Theeuwes, 1992).

However, if the masked color singleton prime captured attention in a top-down-controlled fashion, searching for a visible shape singleton target in Experiment 5B should prevent the masked-priming effect by color singleton primes, because a color singleton would not match the participants' top-down search set for shape-defined targets (Kiss, Jolicœur, Dell'Acqua, \& Eimer, 2008). Restated, if the masked priming effect expected in Experiment $5 \mathrm{~A}$ were to arise due a match between the prime-defining dimension (color) and the top-down search set for color singleton targets, no masked-priming effect by the same color singleton primes would be expected in Experiment 5B, because the search was set for shape singleton targets.

The third aim of Experiment 5 was to confirm that the masked singleton prime exerts its priming effect in the CRT independently of prime visibility. Performance in the prime visibility tests in Experiments 1-4 indicated residual visibility of the singleton primes, which might have been crucial for the masked-priming effect in the CRTs of these experiments. Two measures were taken in Experiment 5 to test this. First, the number of participants was increased, permitting us to median-split them according to their prime visibility test scores and then examine for differences in the masked-priming effects in the CRTs between these two groups. If the masked-priming effects were independent of the prime visibility, priming effects of a similar magnitude would be expected in both groupsthat is, regardless of whether individual prime discrimination performance was above or below the median.

As a further measure for checking that the expected masked-priming effect in the CRTs in Experiment 5 would not depend on prime visibility, we included a con- 

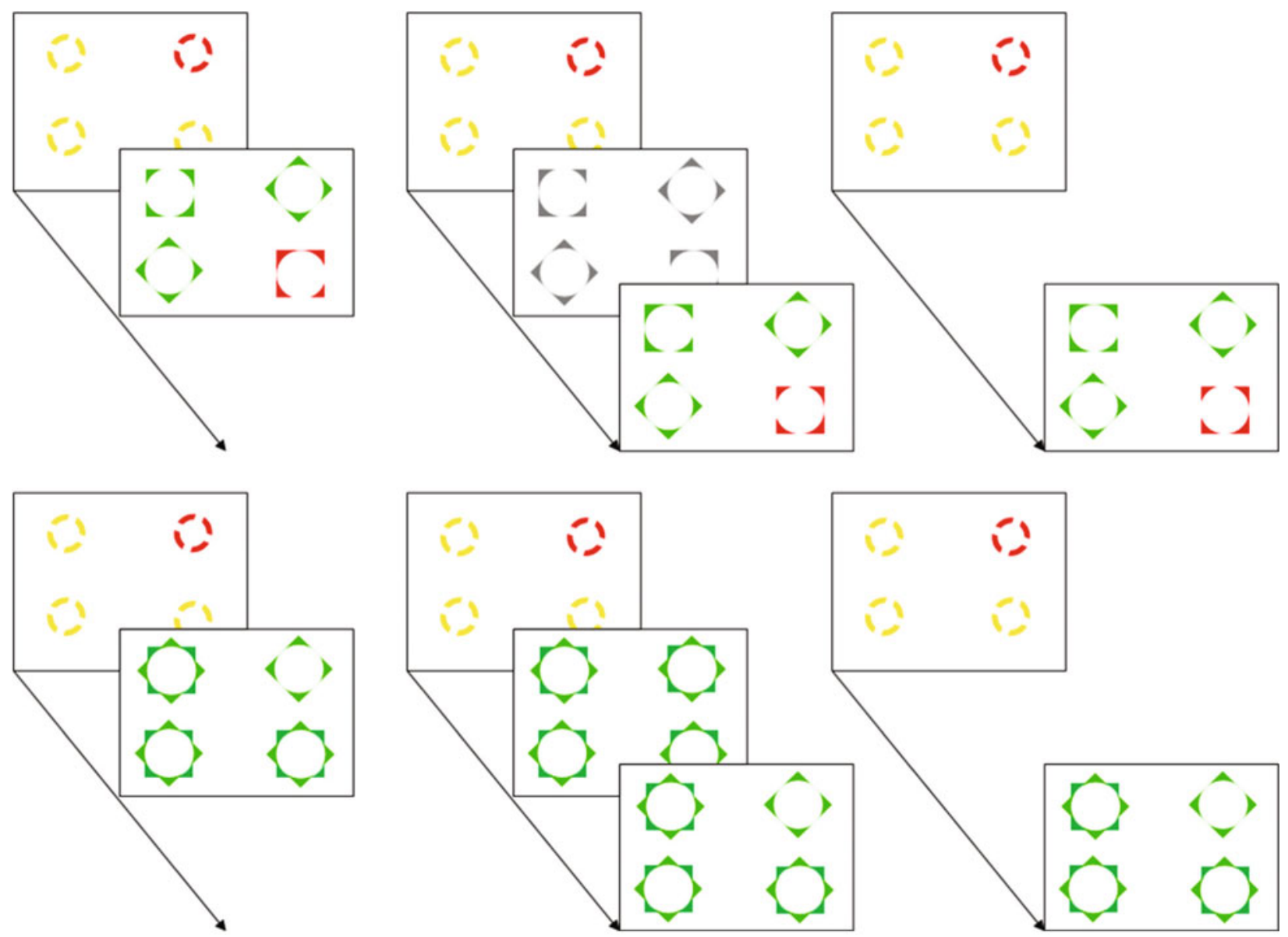

Figure 4. Schematic depiction of the sequence of events in Experiment 5A (upper row) and Experiment 5B (lower row). The first screen, presented for 17 msec, contained circular primes, one of which was a color singleton. In the masked short-interval condition (left column), a mask-target display with a color singleton target (upper row) or a shape singleton target (lower row) was shown, with an interval of $51 \mathrm{msec}$ between prime and target onset. In the masked long-interval condition (central column), a masking display without a singleton target was presented with an interval of $51 \mathrm{msec}$ after prime onset, and for a duration of $150 \mathrm{msec}$, and only then was the singleton target shown together with three masks, yielding a prime-target interval of 200 msec. In the unmasked long-interval condition (right column), the sequence of events was the same, but without the presentation of the intermittent masks. Note that the stimuli are not drawn to scale. See the Method section of Experiment 5 for further details. See online version for color, which in the print version is represented by shades of gray.

trol condition with a visible, unmasked color singleton prime - in addition to two masked conditions, one with a brief (51-msec) and one with a long (200-msec) primetarget interval. In the new, unmasked-priming condition, the prime-target interval was also long $(200 \mathrm{msec})$. The rationale for this manipulation was as follows: If prime singleton visibility were crucial for obtaining maskedpriming effects in the short-interval CRT condition in Experiment 5, no such effects would be expected in either the visible (unmasked) or in the invisible (masked) long prime-target interval conditions. This should be so because an interval of more than $150 \mathrm{msec}$ between an irrelevant color singleton nontarget and a relevant color singleton target has been found to be sufficient for participants to deallocate attention from an irrelevant nontarget if this had initially captured attention (see Kim \& Cave, 1999; Theeuwes, Atchley, \& Kramer, 2000). In the present experiment, rapid deallocation of attention from the irrel- evant prime was reinforced by introducing four possible target locations, with color prime position being nonpredictive of the location of the subsequent shape target (i.e., on $75 \%$ of the trials, the prime was misleading). Thus, if the masked congruence effect reflected prime visibility, we expected zero capture, or a null priming effect, in the visible priming condition, as well as in the long-interval masked-priming condition. (A masked congruence effect in the short-interval condition was to be expected at any rate, at least in Experiment $5 \mathrm{~A}$, since the prime fitted the top-down search sets for color singleton targets and $51 \mathrm{msec}$ would be too short for attentional deallocation.)

However, if the expected masked priming effect in the short-interval CRT conditions in Experiment 5 reflected attentional capture by primes that remained outside the participants' awareness, one would expect a qualitative difference of processing between the invisible (masked) and the visible (unmasked) long-interval conditions (see 
Dixon, 1970; Forster, 1998). The reason is that reactive modification of processing following a misguiding visual prime would be much harder with invisible than with visible stimuli (see Ansorge, Heumann, \& Scharlau, 2002; Cheesman \& Merikle, 1985). Ivanoff and Klein (2003) observed that after initial capture by invisible stimuli, attention could not be deallocated from the position of the capturing stimulus within $300 \mathrm{msec}$ (see also Bauer, Cheadle, Parton, Müller, \& Usher, 2009). Likewise, Ansorge et al. (2002) found that the pace of learning to deallocate attention from an irrelevant singleton was proportional to the singleton's visibility. Thus, if the masked singleton priming effect did not depend on visibility, we expected a priming effect from masked, but not unmasked, prime singletons. Again, this prediction should be borne out at least in Experiment $5 \mathrm{~A}$, where the color singleton prime matched the top-down search set for color singleton targets.

\section{Method}

Participants. Twenty-four participants (19 of them female) participated in Experiment 5A, and 24 different participants (18 of them female) in Experiment 5B. None of them had taken part in the previous experiments. The participants' ages ranged from 18 to 40 years, they all had normal or corrected-to-normal vision and received course credit for their participation.

Stimuli and Procedure. For Experiment 5A, see Figure 4 for the stimuli and procedure. All the stimuli were shown on a computer monitor with a refresh rate of $17 \mathrm{msec}$. A small rectangular fixation aid was shown at the screen center throughout each trial. Trials started with four prime rings $\left(1.3^{\circ}\right.$ in diameter) presented for $17 \mathrm{msec}$ on the corners of an imaginary square around fixation, at an eccentricity of $4.3^{\circ}$. Next, in the two masked-priming conditions (with a short 51msec and a long 200-msec prime-target interval), four angular backward masks (side length of $1.6^{\circ}$ ) were presented for $200 \mathrm{msec}$, with a prime-mask SOA of $51 \mathrm{msec}$. Two of the masks were diamonds, and two were squares; one of the masks was the color-defined target (see below). Since the masks were presented at the same positions as the primes, surrounding the rings (metacontrast masking them), they effectively reduced the visibility of the prime's color (see Ansorge, Becker, \& Breitmeyer, 2009; Ansorge, Kiss, \& Eimer, 2009; Breitmeyer, Ro, \& Singhal, 2004; T. Schmidt, 2002).

In the unmasked condition, the same four angular figures were shown, but with an SOA of $200 \mathrm{msec}$, instead of $51 \mathrm{msec}$, after the primes. This interval is too long for backward masking of the primes by the trailing angular figures (see Alpern, 1953). Therefore, the angular figures did not act as "masks" in the unmasked condition.

For each participant, two out of three colors-red, green, or blue (CIE coordinates, .619/.333, .295/.579, and .151/.107, respectively) - were chosen randomly as the singleton prime colors (e.g., red and green). One of these prime colors (e.g., red) was not used for the coloring of any other stimulus - which is why we term it prime-only color. Out of, altogether, five colors - the remaining potential prime singleton color that was not used for the singleton primes (in the example, blue), plus brown (.439/.470), purple $(.276 / .138)$, turquoise $(.201 / .310)$, and gray $(.281 / .303)$ —one color was randomly selected for the three circular nonsingleton primes on each trial. Out of, altogether, six colors - all of the above colors, with the exception of the prime-only color-two colors were chosen randomly on each trial, one for coloring of the singleton target and another color for the three nonsingleton angular figures.

The colors of targets and masks were selected independently of the prime colors. Prime-only color singleton primes were used on half of the trials. Such "primes" could neither prime the color of the subsequent target (or a mask) nor benefit from priming of their own color by a target (or a mask) on the preceding trial. On the other half of the trials, the color of the prime singleton was sometimes also used for the target or, respectively, the other (nontarget) masks and, so, could have primed the color(s) of the subsequent stimuli or benefited from color priming itself. In all three interval conditions, the positions of the masked color singleton prime and of the visible color singleton target were uncorrelated. As a result, there were $25 \%$ congruent trials, with singleton prime and singleton target at the same position, and $75 \%$ incongruent conditions, with singleton prime and singleton target at different positions.

There were three blocked conditions. On trials in the short-interval masked-priming block, four masks were presented at an SOA of $51 \mathrm{msec}$ after the primes. Two of the masks were squares, and two were diamonds; three of the masks had the same color (nontargets), and one was a color singleton target. Similarly, on the trials in the long-interval masked-priming block, four masks were presented at an SOA of $51 \mathrm{msec}$ after the primes. However, these were all nonsingleton masks of the same color. Only after another $150 \mathrm{msec}$ did all these masks change their colors, with one mask taking on a different color, relative to the other three masks, to become the color singleton target (i.e., the prime-target interval was $200 \mathrm{msec}$ ). Finally, on the trials in the long-interval unmasked condition, no masks were presented $51 \mathrm{msec}$ after prime onset, and the target display, with a color singleton target and three nonsingleton nontargets (as in the masked long-interval condition), appeared only after another $150 \mathrm{msec}$ (with a blank screen).

Again, the participants performed two tasks: the CRT followed by a prime visibility test. Both tasks consisted of three, blocked interval conditions: masked short-interval, masked long-interval, and unmasked long-interval conditions. In each of these blocks, the participants initiated a trial by pressing a central response key with their right-hand index finger. The participants could take a break whenever they chose, simply by postponing the initiation of the next trial. In addition, the participants were encouraged to take a brief break following a prompt presented after every 32 trials.

In the CRT, the participants had to respond to the shape of the color singleton target. Half of the participants had to press the left button for a diamond and the right button for a square, and vice versa for the other half. If the RT on a trial exceeded $1,250 \mathrm{msec}$, a screen was shown (for $750 \mathrm{msec}$ ) asking the participant to respond faster; another screen informed the participants of an incorrect response.

In the prime visibility test, exactly the same prime-target displays were used as in the CRT, but now the participants had to detect one of the two color singleton primes. Initially, the participants were presented with a slow-motion version of the two different color primes, and it was carefully explained to them that they now had to report (in a normally paced version of the event sequence) whether a particular color singleton prime (e.g., a red one) had been presented or not. The participants were also informed that the particular color singleton prime they had to report was present on half of the trials and absent on the other half (in which the alternative prime singleton was shown). The participants were warned that the color of the singleton prime could not be judged on the basis of the color of the singleton target (because their colors were uncorrelated).

For their judgments, the participants pressed the same buttons as in the CRT-however, this time, without any speed stress. They were informed neither about their judgment speed nor about their errors. Whether the right key had to be pressed to report the presence of the searched-for prime color singleton and the left key for its absence, or vice versa, was determined after each (visibility test) trial by means of an instruction display. This trial-by-trial instruction has the advantage of preventing an unwelcome contribution of awareness-independent, "direct" (see Neumann, 1990) response activation to performance on the visibility test. In contrast to Experiments $1-4$, the participants simply gave a two-forced-choice response, rather than additionally rating the confidence (certainty) in their judgment. The participants practiced both tasks prior to data acquisition. Performance in the visibility test was assessed in terms of the SDT measure $d^{\prime}$ - that is, the difference between the $z$-transformed hit rates (here, correct present responses) and the $z$-transformed FA rates (here, incorrect present responses). If there 
was residual prime visibility, $d^{\prime}$ should be significantly greater than zero (i.e., hit rate $>$ FA rate).

Both the CRT and visibility tests consisted of three blocks. Each block consisted of 96 trials, with three repetitions of 2 prime singleton colors $\times 4$ prime locations $\times 4$ target locations. The order of the blocks was balanced across participants, in both the CRT and the visibility test. Including practice, breaks, and instructions, the participants took about 90 min to complete the total of (3 [blocks] $\times$ 2 [tasks] $\times 96$ trials $=) 576$ trials.

For Experiment 5B, see Figure 4 for the stimuli and procedure. The stimuli and procedure were the same as those in Experiment 5A, with the following exceptions. First, on each trial, all four angular figures were color homogeneous and kept their color throughout a trial. Second, the target was defined as the shape singleton among three angular nonsingleton figures. All of the latter were star-shaped (realized by overlaying the diamond and square outlines). By contrast, the shape singleton target was equally likely to be a square or a diamond.

\section{Results}

Experiment 5A: Target CRT. See Figure 5 for the CRT results. Two participants had to be excluded because of an excessive number of errors $(>25 \%)$ in the CRT, and 1 participant's data from the visibility test were lost due to technical error.

The correct mean RTs were subjected to an ANOVA with the within-participants variables of congruence (congruent, incongruent), block (masked short interval, masked long interval, unmasked), and singleton prime type (mask-color singleton prime, prime-only color singleton prime). This ANOVA yielded significant main effects of congruence $[F(1,20)=4.92, p<.05]$ and block $[F(2,40)=5.79, p<.01]$ and a significant congruence $\times$ block interaction $[F(2,40)=3.51, p<.05]$.
Confirming the expected attentional capture effect of the color singleton primes, CRT responses were faster in the congruent than in the incongruent conditions (752 vs. $764 \mathrm{msec}$ ). Also, RTs were shorter in masked shortinterval conditions $(731 \mathrm{msec})$ than in both of the longinterval conditions, the masked $(772 \mathrm{msec})$ and unmasked (771 msec) conditions (both $p \mathrm{~s}<.05$, Bonferroni corrected). Crucially, confirming a qualitative difference between the masked and unmasked conditions, planned comparisons conducted to examine the congruence $\times$ block interaction revealed significantly shorter RTs in the masked congruent than in the masked incongruent conditions, both for short prime-target intervals [719 vs. 743 msec; $t(20)=3.49, p<.01]$ and for long intervals [765 vs. 779 msec; $t(20)=2.04, p<.05]$, but not in the unmasked long-interval conditions $[773 \mathrm{vs} .770 \mathrm{msec}$, $t(20)<1]$. The main effect of cue type and its interactions were all nonsignificant (all $F_{\mathrm{S}}<1$ ).

In addition, we separated our group of participants into halves by their performance on the visibility test. On average, the visibility test indicated better-than-chance performance even in the masked conditions (see below). Yet this was not the case for the 11 participants with a lower than average performance, for whom $d^{\prime}$ was 0.006 in the masked short-interval condition and 0.008 for the masked long-interval condition (comparisons against zero; both ts $<1$ ).

In line with the assumption that the masked singleton priming effect did not depend on awareness of the prime singletons, for the participants with a lower than average performance, planned comparisons confirmed congruence effects in both the masked short-interval condition

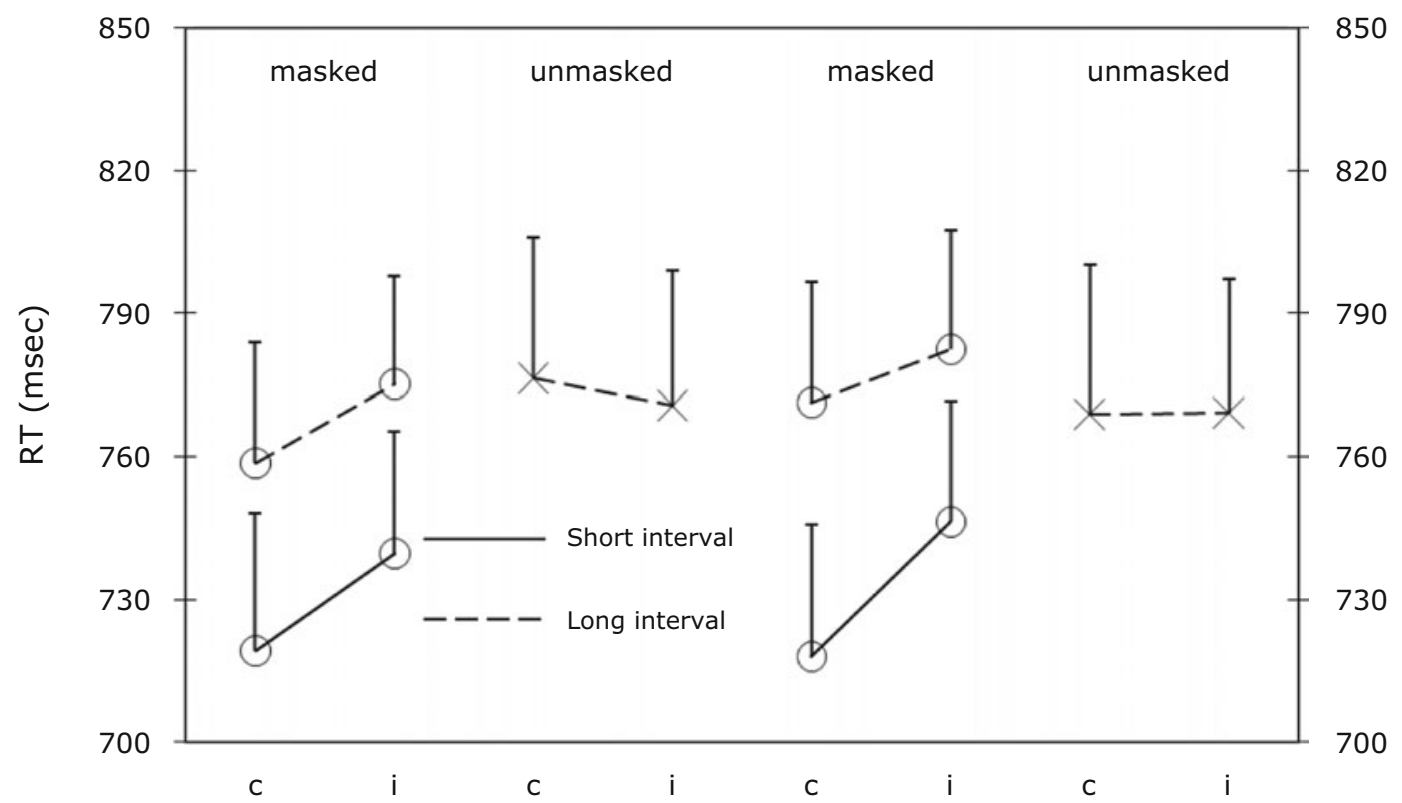

Figure 5. Response times (RTs, in milliseconds) as a function of congruence (c, congruent; i, incongruent), masking (masked, graphs on the far left and second from right; unmasked, graphs second from left and on the far right), prime-target interval (drawn lines, $51 \mathrm{msec}$; broken lines, $200 \mathrm{msec}$ ), and prime type (target- or masksimilar primes on the right; prime-only color singletons on the left) in Experiment $5 \mathrm{~A}$ (data for 21 participants). Bars indicate standard errors. 
[705 vs. $801 \mathrm{msec}$ for congruent and incongruent trials, respectively; $t(10)=2.38, p<.05]$ and the masked longinterval condition [ 805 vs. $821 \mathrm{msec} ; t(10)=2.10, p<$ $.05]$, but not in the unmasked long-interval conditions (841 vs. $842 \mathrm{msec} ; t<1$ ).

An ANOVA of the PEs with the same factors yielded only a significant main effect of prime type $[F(1,20)=$ 4.76, $p<.05$ ], with lower performance with prime-only singleton color primes $(\mathrm{PE}=6.3 \%)$ than with masksimilar color singleton primes $(\mathrm{PE}=4.8 \%)$. All other main effects (all $F_{\mathrm{s}}<1$ ) and interactions (all $F_{\mathrm{s}}<3.20$, all $p \mathrm{~s}>.09$ ) were nonsignificant.

Experiment 5A: Prime visibility. Pairwise comparison by $t$ tests confirmed that primes were more visible in the unmasked conditions $\left(d^{\prime}=0.56\right)$ than in the masked long-interval $\left(d^{\prime}=0.10\right)$ and masked short-interval $\left(d^{\prime}=\right.$ $0.17)$ conditions [both $t \mathrm{~s}(20)>6.60$, both $p \mathrm{~s}<.01]$. Although $d^{\prime}$ was not very high in any of the conditions, planned comparisons with zero revealed better-thanchance performance in all the blocked conditions (all $t \mathrm{~s}>$ 2.00 , all $p \mathrm{~s}<.05$ ). Confirming a congruence effect of primes remaining outside of participants' awareness, the intercept of linear regressions that regressed individual RT congruence effects (incongruent RT - congruent RT) on individual prime visibility scores $\left(d^{\prime}\right)$ were significant at short $[t(20)=2.71, p<.05]$ and long $[t(20)=2.13$, $p<.05]$ masked-interval conditions.

Experiment 5B: Target CRT. See Figure 6 for the CRT results. One participant had to be excluded because of an excessive number of errors ( $>25 \%$ ).

The ANOVA of the correct RTs revealed a significant main effect of block $[F(2,44)=7.67, p<.01]$. Again,
RTs were shorter in the masked short-interval conditions (693 msec) than in both of the long-interval conditions, the masked (748 msec) and unmasked (729 msec) conditions (both $p \mathrm{~s}<.05$, Bonferroni corrected). In line with the assumption that the masked prime color singletons captured attention in a top-down contingent fashion, the main effect of congruence was nonsignificant in Experiment 5B (723 vs. $724 \mathrm{msec}$ for congruent and incongruent trials, respectively; $F<1$ ). All interactions were nonsignificant, too (all $F_{\mathrm{s}}<1.40$, all $p \mathrm{~s}>.27$ ).

The 12 participants with below-average scores were again not significantly above chance (as a group) in the masked conditions of the visibility test, with $d^{\prime}=0.089$ in the short-interval condition $[t(11)=1.80, p=.10]$ and $d^{\prime}=0.049$ in the long-interval condition $(t<1.00)$. These participants, however, showed no significant priming effect in any of the CRT conditions. Congruent RTs were not significantly shorter than incongruent RTs in the masked short-interval condition (709 vs. $704 \mathrm{msec} ; t<1$ ), in the masked long-interval condition (751 vs. $749 \mathrm{msec}$; $t<1$ ), or in the unmasked long-interval condition [719 vs. $728 \mathrm{msec} ; t(11)=1.13, p<.27]$. An ANOVA of the error rates suggested that the task-irrelevant color singletons were actively inhibited: The only significant main effect was that of congruence $[F(1,22)=44.85, p<.01]$, with better performance in the incongruent than in the congruent conditions $(4.2 \%$ vs. $6.5 \%)$. That is, a target presented at the position of the irrelevant singleton prime was associated with a processing cost, perhaps reflecting active inhibition of the preceding singleton.

Experiment 5B: Prime visibility. Pairwise comparison by $t$ tests confirmed that primes were more visible in

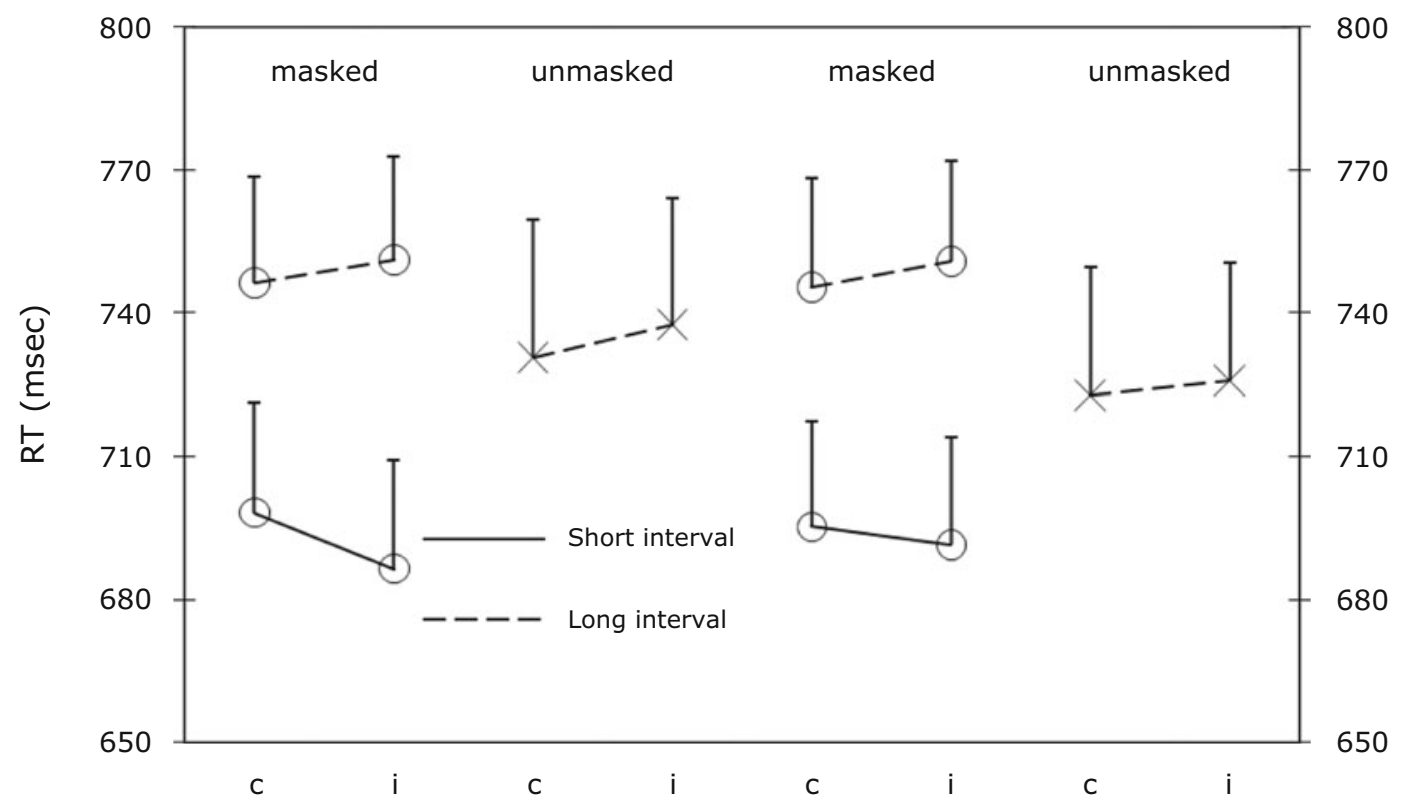

Figure 6. Response times (RTs, in milliseconds) as a function of congruence (c, congruent; $i$, incongruent), masking (masked, graphs on the far left and second from right; unmasked, graphs second from left and on the far right), prime-target interval (drawn lines, $51 \mathrm{msec}$; broken lines, $200 \mathrm{msec}$ ), and prime type (target- or masksimilar primes on the right; prime-only color singletons on the left) in Experiment 5B (data for 21 participants). Bars indicate standard errors. 
the unmasked conditions $\left(d^{\prime}=2.24\right)$ than in the masked long-interval $\left(d^{\prime}=0.54\right)$, and masked short-interval $\left(d^{\prime}=\right.$ $0.62)$ conditions [both $t \mathrm{~s}(20)>6.80$, both $p \mathrm{~s}<.01] . d^{\prime}$ was very high in the unmasked conditions, and it was still substantial in the masked conditions, so that all planned comparisons with zero revealed better-than-chance performance in the different blocked conditions (all $t \mathrm{~s}>2.90$, all $p \mathrm{~s}<.01)$. Of course, since no significant congruence effects were found in the first place, there was also no significant intercept of linear regressions that regressed individual RT congruence effects (incongruent RT - congruent RT) on individual prime visibility scores $\left(d^{\prime}\right)$ with short and long masked-interval conditions (both $t \mathrm{~s}<1$ ).

\section{Discussion}

In Experiment 5, we examined attentional capture by masked singleton primes under conditions in which the primes were prevented from (pre)activating the response required by the subsequent target. Despite this, a congruent masked singleton prime (appearing at the position of the upcoming visible color singleton target) facilitated RTs to the target, in comparison with an incongruent prime (presented at a nontarget position). This masked priming effect was similar in magnitude whether the prime shared the color of the visible target or the (nontarget) masks or whether its color was never used for any of the visible stimuli. Given this, color priming (e.g., Belopolsky, Schreij, \& Theeuwes, 2010; Maljkovic \& Nakayama, 1994) by the masked singletons (from the preceding trial) cannot explain the congruence effect generated by the masked primes (on the current trial). Thus, taken together, the pattern of results indicates that the masked priming effect in Experiment 5 is attributable to the singleton prime summoning ("capturing") attention to its position.

Experiment 5 also suggests that visibility of the masked color singleton prime was not responsible for the maskedpriming effect. With an unmasked visible prime, we observed no singleton capture. This was the case even in conditions in which the visible prime matched the topdown search set. The reason for this lack of priming with unmasked primes is that the relatively long prime-target interval (of $200 \mathrm{msec}$ ) gave the participants sufficient time to deallocate attention away from the attention-capturing, but likely misguiding, singleton, so that a significant priming effect was no longer observed (see Kim \& Cave, 1999). Yet, with masked primes in the same interval conditions, we did find a priming effect, indicating that the participants were unable to deallocate attention away from the masked singletons when the prime was invisible (see Ivanoff $\&$ Klein, 2003). This qualitative difference in processing between masked and unmasked prime conditions strongly suggests that the masked priming effect was independent of the masked prime's visibility (see Dixon, 1970).

Consistent with this, even when only those participants in Experiment 5A who were unable to discern the masked prime singletons in the visibility test are considered, they still showed a masked-priming effect, indicative of attentional capture, in the CRT. Thus, again, it would appear unlikely that residual prime visibility is crucial for generating this effect.
Furthermore, we found evidence that the maskedpriming effect was top-down contingent. In Experiment 5A, the participants searched for a color singleton target. Under these conditions, the masked color singleton prime matched the top-down controlled set (see Folk et al., 1992), which specified search for a color-defined singleton target, and we did observe a significant maskedpriming effect. By contrast, in Experiment 5B, the participants had to search for a shape-defined singleton target, so that the masked color singleton prime did not match the top-down search set. Under these conditions, we failed to observe a masked-priming effect. This result differs from the attentional capture effect by clearly visible color singletons when participants search for a shape singleton (see Bacon \& Egeth, 1994), and this difference between the previous and present findings might, at least in part, reflect the specifics of attentional capture by masked stimuli.

Interestingly, as in Ivanoff and Klein's (2003) study, in Experiment 5A, a task-relevant subliminal stimulus was hard to ignore, giving rise to a congruence effect. However, such a qualitative processing difference between visible and invisible primes was no longer observed with the task-irrelevant subliminal prime in Experiment 5B; that is, such a prime could be ignored as efficiently as a visible prime.

Another, unexpected finding of Experiment 5 deserves discussion: Prime color singleton discriminabilty (in the visibility test) was higher in Experiment $5 \mathrm{~B}$ than in Experiment 5A. This finding may well be owing to the greater color homogeneity of the angular masks in Experiment 5B - as compared with Experiment 5A, in which either one singleton color target was presented at a primetarget interval of $51 \mathrm{msec}$, or all of the masks changed color after $150 \mathrm{msec}$ to give way to the color singleton target display. More research is needed, however, to fully understand this finding.

\section{GENERAL DISCUSSION}

In the present study, we confirmed that singletons remaining outside participants' awareness were processed to some extent. This was reflected in a congruence effect in the RTs to visible targets - that is, a difference in performance dependent on whether a masked prime singleton was congruent or incongruent in position with a subsequently presented, visible target. Subjectively, the masked prime was never seen. Objectively, though, the participants were above chance in locating (at least) the featurepresent primes or in discriminating prime singleton color. In Experiments 1-4, however, the correlations between the individual prime visibility scores and congruence effects were low and nonsignificant. Thus, prime visibility was not a good predictor of the prime's congruence effect (see Greenwald et al., 1996; Holender \& Duscherer, 2004). In Experiment 5, splitting the sample of participants into halves by their performance on the prime visibility test, we found that even participants who were unable to discriminate the primes with above-chance accuracy (in an objective test) did show a significant congruence effect. In 
addition, qualitative differences between prime processing in visible (unmasked) and masked conditions suggest that masked priming effects were independent of visual awareness, at least in Experiment 5.

The finding, in the present study, of a congruence effect produced by masked singletons is in line with the conclusions of Zhaoping (2008), although also ruling out some uncertainties that still remained after Zhaoping's (2008) study. First, we observed a congruence effect of masked prime singletons regardless of whether the masked prime could (e.g., in Experiment 1) or could not (e.g., in Experiment 2) be found by visually searching for the targetdefining feature. In this respect, the effect seemed to depend on mere feature contrasts (see Parkhurst et al., 2002). However, second, by demonstrating the effect under conditions of metacontrast masking and finding an even stronger effect of singletons defined by visual image features, it is relatively certain that part of the effect is brought about by feature salience computed in V1 $(\mathrm{Li}, 2002)$ and that the effect generalizes to natural viewing conditions, as proposed by Zhaoping (2008) in the first place. The present findings are therefore in line with the assumption that mere visual feature differences, rather than awareness of a pop-out stimulus, can account for singleton-driven effects. This has been assumed all along in computational theories of feature differences driving covert and overt attentional selection (see Itti \& Koch, 2001; Parkhurst et al., 2002).

The present results also provide (at least some) pointers regarding the question of which of several conceivable effects of the masked singletons can account for the congruence effect. Of note, there was a masked congruence effect even in conditions in which the singleton was defined in a way other than the target (e.g., by feature absence when the target was defined by feature presence, or by a color that the target and the mask could never take on). Consequently, the singleton effect may reflect stimulusdriven processes (see Donk \& van Zoest, 2008; van Zoest et al., 2004).

However, the singleton effect also appears to reflect goal-dependent processing. Importantly, we found that even with a very short interval between the prime and target, a masked singleton defined in another feature dimension (here, color) than that of the target (here, shape) was successfully inhibited in Experiment 5A (see also Müller, Krummenacher, Geyer, \& Zehetleitner, 2009). In addition, in all of the present experiments, it was theoretically possible for participants to willingly search for singleton targets (see Bacon \& Egeth, 1994; Leber \& Egeth, 2006). On this basis alone, we cannot rule out that goal settings as a top-down factor were a crucial precondition for the priming effects engendered by the masked singletons.

It is difficult to decide to what extent the singleton effect of the masked primes in Experiments 1-4 was due to attentional capture, rather than to response activation. Many prior studies have interpreted masked priming effects as exclusively, or mainly, reflecting response activation processes (see Leuthold \& Kopp, 1998; Neumann, 1990; Neumann \& Klotz, 1994). Recent evidence, how- ever, has shown that cognitive operations can be likewise primed by masked stimuli and, most importantly, that a masked prime can capture attention (see Ansorge, Kiss, \& Eimer, 2009; Bodis-Wollner, 2008; Scharlau \& Ansorge, 2003; Scharlau \& Neumann, 2003, F. Schmidt \& Schmidt, 2010). We contend that it is most likely that the effects in Experiments 1-4 reflected both attentional capture and motor response activation. First, theoretically, discriminating motor activation from attentional capture is not well motivated if one conceives of the shifting of attention as the specification of (saccadic) motor parameters (see Rizzolatti, Riggio, Dascola, \& Umiltà, 1987). Second, empirically, when isolating the attentional capture effect of the prime in Experiment 5, we observed a sharp decline in the congruence effect, in comparison with Experiments $1-4$, and the congruence effect was no longer influenced by the interval between the prime and target. Arguably, therefore, it is very likely that processes such as response activation by the masked prime, in addition to attentional capture, contributed to the congruence effects in Experiments 1-4.

In two respects, the present findings are also interesting vis-à-vis previous studies of masked priming. First, we demonstrated that masked-priming effects can partly depend on the processing of the masked prime's singleton status. To our knowledge, this possibility has so far not been considered, although several masked-priming effects lend themselves to such an interpretation (see Ansorge, 2004; Leuthold \& Kopp, 1998; Neumann \& Klotz, 1994). Second, we consistently found above-chance performance in a prime visibility test under conditions in which others found no such evidence (see Ansorge, 2003; Ansorge \& Neumann, 2005; Leuthold \& Kopp, 1998; Neumann \& Klotz, 1994). In Experiments 1-4, one reason for the difference is probably the amount of practice that participants had: The same 11 participants discriminated the prime locations on, altogether, 960 trials (not counting other, similar experiments in which these participants had taken part). Practice is a known factor facilitating performance on visibility tests under masking conditions (Wolford et al., 1988). Another reason for the above-chance accuracy in the visibility tests in Experiments 1-4, as compared with prior studies, could lie in the fact that we used a location discrimination task. If the prime was on the right, the participants had to judge "right"'; and if it was on the left, the participants had to judge "left." This is a 2:2 task in which two stimuli (left vs. right primes) map onto two judgment alternatives (left vs. right judgments). As compared with this, previous visibility tasks may have been somewhat more demanding, because 3 stimuli (congruent trials, incongruent trials, and neutral trials without a prime) were mapped onto two judgments (3:2 mapping): judgments about the presence of a prime, requiring the same judgment for congruent and incongruent conditions, and an alternative no-prime judgment in neutral conditions. This arguably more difficult mapping could have underestimated visibility measures such as $d^{\prime}$ by a factor of $\sqrt{ } 2$ (Macmillan \& Creelman, 2005). Support for this assumption is provided by a systematic comparison of mapping rules that varied in 
difficulty (Ansorge et al., 2007). Finally, in Experiment 5, improvement of performance in the prime visibility test was evident under conditions with a more balanced (here, color-homogeneous) masking display. Under these conditions, subtle perceptual consequences of the prime, such as flicker, were probably easier to detect.

In closing, we emphasize that we do not consider processing of singletons in general and singleton capture in particular to be restricted to early and awarenessindependent stages of visual processing. Independence of awareness could be a characteristic for some stimulusdriven singleton or salience effects occurring early in the course of visual processing (see Donk \& van Zoest, 2008; Kim \& Cave, 1999). In addition, with a task, or search, set for the singletons being set up in advance of the masked primes, awareness-independent singleton effects could be partly goal dependent, too (see Ansorge, Becker, \& Breitmeyer, 2009; Neumann, 1990). Realistically, some singleton effects, including stimulus-driven effects, could also crucially depend on singleton awareness. Frey, Honey, and König (2008), for instance, found that local red-green contrasts affected eye fixations only while participants viewed images of rain forests, but not, for example, while viewing images of fractals. Such results suggest a role for the surrounding feature context (defined by spatial frequencies or orientations) in color singleton effects. To the extent that context effects occur late during visual processing and that they require awareness (see Lamme \& Roelfsema, 2000; Lamme, Zipser, \& Spekreijse, 2002; Zipser, Lamme, \& Schiller, 1996), the corresponding singleton effects could be crucially dependent on awareness, too.

\section{AUTHOR NOTE}

This research was supported by SERC (U.K.) Grant GR/H/54966 and German Research Foundation (DFG) Grants FOR480 and EC142 (Excellence Cluster 'CoTeSys') to H.J.M., and by DFG Grants AN 393/2-1 to U.A. and AN 393/5-1 to U.A., Werner Klotz, and Ingrid Scharlau. Correspondence concerning this article should be addressed to U. Ansorge, Fakultät für Psychologie, Liebiggasse 5, A-1010 Wien, Austria (e-mail: ulrich.ansorge@univie.ac.at).

\section{REFERENCES}

Alpern, M. (1953). Metacontrast. Journal of the Optical Society of America, 43, 648-657.

Ansorge, U. (2003). Asymmetric influences of temporally vs. nasally presented masked visual information: Evidence for collicular contributions to nonconscious priming effects. Brain \& Cognition, 51, 317-325.

ANSORGE, U. (2004). Top-down contingencies of nonconscious priming revealed by dual-task interference. Quarterly Journal of Experimental Psychology, 57A, 1123-1148.

Ansorge, U., Becker, S. I., \& Breitmeyer, B. G. (2009). Revisiting the metacontrast dissociation: Comparing sensitivity across different measures and tasks. Quarterly Journal of Experimental Psychology, 62, 286-309.

Ansorge, U., Breitmeyer, B. G., \& Becker, S. I. (2007). Comparing sensitivity across different processing measures under metacontrast masking conditions. Vision Research, 47, 3335-3349.

Ansorge, U., Heumann, M., \& Scharlau, I. (2002). Influences of visibility, intentions, and probability in a peripheral cuing task. Consciousness \& Cognition, 11, 528-545.

Ansorge, U., Kiss, M., \& EIMER, M. (2009). Goal-driven attentional capture by invisible colors: Evidence from event-related potentials. Psychonomic Bulletin \& Review, 16, 648-653.

Ansorge, U., \& Neumann, O. (2005). Intentions determine the effects of invisible metacontrast-masked primes: Evidence for top-down contingencies in a peripheral cueing task. Journal of Experimental Psychology: Human Perception \& Performance, 31, 762-777.

BaCon, W. F., \& Egeth, H. E. (1994). Overriding stimulus-driven attentional capture. Perception \& Psychophysics, 55, 485-496.

Bauer, F., Cheadle, S. W., Parton, A., Müller, H. J., \& Usher, M. (2009). Gamma flicker triggers attentional selection without awareness. Proceedings of the National Academy of Sciences, 106, 16661671.

Belopolsky, A.V., SchreiJ, D., \& Theeuwes, J. (2010).What is topdown about contingent capture? Attention, Perception, \& Psychophysics, 72, 326-341

Bergen, J. R., \& Julesz, B. (1983). Parallel versus serial processing in rapid pattern discrimination. Nature, 303, 696-698.

Bodis-Wollner, I. (2008). Pre-emptive perception. Perception, 37, 462-468.

BReitmeyer, B. G. (1984). Visual masking: An integrative approach. New York: Oxford University Press.

Breitmeyer, B. G., \& Ogmen, H. (2006). Visual masking: Time slices through conscious and unconscious vision. Oxford: Oxford University Press.

Breitmeyer, B. G., Ro, T., \& Singhal, N. S. (2004). Unconscious color priming occurs at stimulus- not percept-dependent levels of processing. Psychological Science, 15, 198-202.

Burnham, B. R. (2007). Displaywide visual features associated with a search display's appearance can mediate attentional capture. Psychonomic Bulletin \& Review, 14, 392-422.

Burnham, B. R., \& Neely, J. H. (2008). A static color discontinuity can capture spatial attention when the target is an abrupt-onset singleton. Journal of Experimental Psychology: Human Perception \& Performance, 34, 831-841.

Cheesman, J., \& Merikle, P. M. (1985). Word recognition and consciousness. In D. Besner, T. G. Waller, \& G. E. MacKinnon (Ed.), Reading research: Advances in theory and practice (pp. 311-352). New York: Academic Press.

Cole, G. G., Kentridge, R. W., \& Heywood, C. A. (2005). Object onset and parvocellular guidance of attentional allocation. Psychological Science, 16, 270-274.

Dixon, N. F. (1970). Subliminal perception: The nature of a controversy. New York: McGraw-Hill.

DonK, M., \& van Zoest, W. (2008). Effects of salience are short-lived. Psychological Science, 19, 733-739.

Dorfman, D. D. (1982). RSCORE II. In J. A. Swets \& R. M. Pickett (Eds.), Evaluation of diagnostic systems: Methods from signal detection theory (pp. 212-232). New York: Academic Press.

Dorfman, D. D., \& Alf, E., JR. (1969). Maximum likelihood estimation of parameters of signal detection theory and determination of confidence intervals-rating method data. Journal of Mathematical Psychology, 6, 487-496.

Fehrer, E., \& RAAB, D. (1962). Reaction time to stimuli masked by metacontrast. Journal of Experimental Psychology, 62, 143-147.

Folk, C. L., Remington, R. W., \& Johnston, J. C. (1992). Involuntary covert orienting is contingent on attentional control settings. Journal of Experimental Psychology: Human Perception \& Performance, 18, 1030-1044.

Forster, K. I. (1998). The pros and cons of masked priming. Journal of Experimental Psychology: Learning, Memory, \& Cognition, 10, 680-698.

Frey, H.-P., Honey, C., \& KöNIG, P. (2008). What's color got to do with it? The influence of color on visual attention in different categories. Journal of Vision, 8(14, Art. 6), 1-17

Green, D. M., \& Swets, J. A. (1966). Signal detection theory and psychophysics. New York: Wiley.

Greenwald, A. G., Draine, S. C., \& Abrams, R. L. (1996). Three cognitive markers of unconscious semantic activation. Science, 273, 1699-1702.

Holender, D. (1986). Semantic activation without conscious identification in dichotic listening, parafoveal vision, and visual masking: A survey and appraisal. Behavioral \& Brain Sciences, 9, 1-66. 
Holender, D., \& Duscherer, K. (2004). Unconscious perception: The need for a paradigm shift. Perception \& Psychophysics, 66, 872-881.

IтTI, L., \& КосH, C. (2001). Computational modelling of visual attention. Nature Reviews Neuroscience, 2, 4-11.

IvanofF, J., \& KleIN, R. M. (2003). Orienting of attention without awareness is affected by measurement-induced attentional control settings. Journal of Vision, 3, 32-40.

JONIDES, J. (1981). Voluntary versus automatic control over the mind's eye's movement. In J. Long \& A. Baddeley (Eds.), Attention and performance IX (pp. 187-203). Hillsdale, NJ: Erlbaum.

KIM, M.-S., \& CAVE, K. R. (1999). Top-down and bottom-up attentional control: On the nature of interference from a salient distractor. Perception \& Psychophysics, 61, 1009-1023.

Kiss, M., Jolicceur, P., Dell'Acqua, R., \& Eimer, M. (2008). Attentional capture by visual singletons is mediated by top-down task set: New evidence from the N2pc component. Psychophysiology, 45, 1013-1024.

Klotz, W., \& Neumann, O. (1999). Motor activation without conscious discrimination in metacontrast masking. Journal of Experimental Psychology: Human Perception \& Performance, 25, 976-992.

Kolers, P. (1963). Some differences between real and apparent visual movement. Vision Research, 3, 191-206.

Lamme, V. A. F., \& Roelfsema, P. R. (2000). The distinct modes offered by feedforward and recurrent processing. Trends in Neurosciences, 23, 571-579.

Lamme, V. A. F., Zipser, K., \& SpekreiJse, H. (2002). Masking interrupts figure-ground signals in V1. Journal of Cognitive Neuroscience, 14, 1044-1053.

LAMY, D., \& ZoARIS, L. (2009). Task-irrelevant stimulus salience affects visual search. Vision Research, 49, 1472-1480.

Leber, A. B., \& Egeth, H. E. (2006). It's under control: Top-down strategies can override attentional capture. Psychonomic Bulletin \& Review, 13, 132-138.

Leuthold, H., \& KopP, B. (1998). Mechanisms of priming by masked stimuli: Inferences from event-related brain potentials. Psychological Science, 9, 263-269.

LI, Z. (2002). A saliency map in primary visual cortex. Trends in Cognitive Sciences, 6, 9-16.

Macmillan, N. A., \& Creelman, C. D. (2005). Detection theory: A user's guide (2nd ed.). Mahwah, NJ: Erlbaum.

Maljkovic, V., \& Nakayama, K. (1994). Priming of pop-out: I. Role of features. Memory \& Cognition, 22, 657-672.

McCormick, P. A. (1997). Orienting attention without awareness. Journal of Experimental Psychology: Human Perception \& Performance, 23, $168-180$.

Mulckhuyse, M., Talsma, D., \& Theeuwes, J. (2007). Grabbing attention without knowing: Automatic capture of attention by subliminal spatial cues. Visual Cognition, 15, 779-788.

Müller, H. J., Krummenacher, K., Geyer, T., \& Zehetleitner, M. (2009). Attentional capture by salient color singleton distractors is modulated by top-down dimensional set. Journal of Experimental Psychology: Human Perception \& Performance, 35, 1-16.

Müller, H. J., \& Rabbitt, P. M. A. (1989). Reflexive and voluntary orienting of visual attention: Time course of activation and resistance to interruption. Journal of Experimental Psychology: Human Perception \& Performance, 15, 315-330.

Naccache, L., \& Dehaene, S. (2001). Unconscious semantic priming extends to novel unseen stimuli. Cognition, 80, 215-229.

Neumann, O. (1990). Direct parameter specification and the concept of perception. Psychological Research, 52, 207-215.

Neumann, O., \& Klotz, W. (1994). Motor responses to nonreportable, masked stimuli: Where is the limit of direct parameter specification? In C. Umiltà \& M. Moscovitch (Eds.), Attention and performance $X V$ : Conscious and nonconscious information processing (pp. 123-150). Cambridge, MA: MIT Press.

Parkhurst, D., LaW, K., \& Niebur, E. (2002). Modelling the role of salience in the allocation of overt visual attention. Vision Research, 42, 107-123.

Posner, M. I. (1980). Orienting of attention. Quarterly Journal of Experimental Psychology, 32A, 3-25.

ReINGOLD, E. M. (2004). Unconscious perception and the classic dis- sociation paradigm: A new angle? Perception \& Psychophysics, 66, 882-887.

Reingold, E. M., \& Merikle, P. M. (1988). Using direct and indirect measures to study perception without awareness. Perception \& Psychophysics, 44, 563-575.

Rizzolatti, G., Riggio, L., Dascola, I., \& Umiltà, C. (1987). Reorienting attention across the horizontal and vertical meridians: Evidence in favor of a premotor theory of attention. Neuropsychologia, 25, 31-40.

SCHARLAU, I. (2002). Leading, but not trailing, primes influence temporal order perception: Further evidence for an attentional account of perceptual latency priming. Perception \& Psychophysics, 64, 1346-1360.

SCHARLAU, I., \& ANSORGE, U. (2003). Direct parameter specification of an attention shift: Evidence from perceptual latency priming. Vision Research, 43, 1351-1363.

Scharlau, I., \& Neumann, O. (2003). Temporal parameters and time course of perceptual latency priming. Acta Psychologica, 113, 185203.

Schmidt, F., \& Schmidt, T. (2010). Feature-based attention to unconscious shapes and colours. Attention, Perception, \& Psychophysics, 72, 1480-1494.

Schmidt, T. (2002). The finger in flight: Real-time motor control by visually masked color stimuli. Psychological Science, 13, 112-117.

Schmidt, T., \& Vorberg, D. (2006). Criteria for unconscious cognition: Three types of dissociation. Perception \& Psychophysics, 68, 489-504.

Snodgrass, M., Bernat, E., \& Shevrin, H. (2004). Unconscious perception: A model-based approach to method and evidence. Perception \& Psychophysics, 66, 846-867.

Steglich, C., \& Neumann, O. (2000). Temporal, but not spatial, context modulates a masked prime's effect on temporal order judgment, but not on response latency. Psychological Research, 63, 36-47.

Theeuwes, J. (1992). Perceptual selectivity for color and form. Perception \& Psychophysics, 51, 599-606.

Theeuwes, J. (2004). Top-down strategies cannot override attentional capture. Psychonomic Bulletin \& Review, 11, 65-70.

Theeuwes, J., Atchley, P., \& Kramer, A. F. (2000). On the time course of top-down and bottom-up control of visual attention. In S. Monsell \& J. Driver (Eds.), Control of cognitive processes: Attention and performance XVIII (pp. 105-125). Cambridge, MA: MIT Press.

Treisman, A., \& GoRmican, S. (1988). Feature analysis in early vision: Evidence from search asymmetries. Psychological Review, 95, 15-48.

Treisman, A., \& Souther, J. (1985). Search asymmetry: A diagnostic for preattentive processing of separable features. Journal of Experimental Psychology: General, 114, 285-310.

van Zoest, W., Donk, M., \& Theeuwes, J. (2004). The role of stimulusdriven and goal-driven control in saccadic visual selection. Journal of Experimental Psychology: Human Perception \& Performance, 30, 746-759.

Vorberg, D., Mattler, U., Heinecke, A., Schmidt, T., \& Schwarzвасн, J. (2003). Different time courses for visual perception and action priming. Proceedings of the National Academy of Sciences, 100, 6275-6280.

Wolford, G., MarchaK, F., \& Hughes, H. (1988). Practice effects in backward masking. Journal of Experimental Psychology: Human Perception \& Performance, 14, 101-112.

YANTIS, S., \& EGETH, H. E. (1999). On the distinction between visual salience and stimulus-driven attentional capture. Journal of Experimental Psychology: Human Perception \& Performance, 25, 661-676.

ZHAOPING, L. (2005). The primary visual cortex creates a bottom-up saliency map. In L. Itti, G. Rees, \& J. K. Tsotsos (Eds.), Neurobiology of attention (pp. 570-575). Amsterdam: Elsevier.

ZHAOPING, L. (2008). Attention capture by eye of origin singletons even without awareness-A hallmark of a bottom-up saliency map in the primary visual cortex. Journal of Vision, 8(5), 1-18.

Zipser, K., Lamme, V. A., \& Schiller, P. H. (1996). Contextual modulation in primary visual cortex. Journal of Neuroscience, 16, 73767389.

(Manuscript received September 2, 2009; revision accepted for publication June 14, 2010.) 